\title{
Nonexistence of Positive Supersolutions of Nonlinear Biharmonic Equations without the Maximum Principle
}

\author{
Marius Ghergu*and Steven D. Taliaferro ${ }^{\dagger \ddagger}$
}

\begin{abstract}
We study classical positive solutions of the biharmonic inequality$$
-\Delta^{2} v \geq f(v)
$$

in exterior domains in $\mathbb{R}^{n}$ where $f:(0, \infty) \rightarrow(0, \infty)$ is continuous function. We give lower bounds on the growth of $f(s)$ at $s=0$ and/or $s=\infty$ such that inequality (0.1) has no $C^{4}$ positive solution in any exterior domain of $\mathbb{R}^{n}$. Similar results were obtained by Armstrong and Sirakov [Nonexistence of positive supersolutions of elliptic equations via the maximum principle, Comm. Partial Differential Equations 36 (2011) 2011-2047] for $-\Delta v \geq f(v)$ using a method which depends only on properties related to the maximum principle. Since the maximum principle does not hold for the biharmonic operator, we adopt a different approach which relies on a new representation formula and an a priori pointwise bound for nonnegative solutions of $-\Delta^{2} u \geq 0$ in a punctured neighborhood of the origin in $\mathbb{R}^{n}$.
\end{abstract}

\section{Introduction}

Using a method which depends only on properties related to the maximum principle, Armstrong and Sirakov [1] proved the following two nonexistence result for positive solutions of

$$
-\Delta v \geq f(v)
$$

in exterior domains in $\mathbb{R}^{n}$.

Theorem 1.1 (Armstrong and Sirakov [1]). Assume that $n \geq 3$ and the nonlinearity $f:(0, \infty) \rightarrow$ $(0, \infty)$ is continuous and satisfies

$$
\liminf _{s \rightarrow 0^{+}} \frac{f(s)}{s^{1+\frac{2}{n-2}}}>0 .
$$

Then the inequality (1.1) has no positive solution in any exterior domain of $\mathbb{R}^{n}$.

The exponent $1+\frac{2}{n-2}$ in (1.2) is optimal because, as pointed out in [1], for each constant $\lambda>1+\frac{2}{n-2}$ there exists a positive constant $C$ such that a solution of $-\Delta v=v^{\lambda}$ in $\mathbb{R}^{n} \backslash\{0\}$, which tends to zero as $|y| \rightarrow \infty$, is $v(y)=C|y|^{\frac{-2}{\lambda-1}}$.

\footnotetext{
${ }^{*}$ School of Mathematical Sciences, University College Dublin, Belfield, Dublin 4, Ireland; marius.ghergu@ucd.ie

${ }^{\dagger}$ Mathematics Department, Texas A\&M University, College Station, TX 77843-3368; stalia@math.tamu.edu

${ }^{\ddagger}$ Corresponding author, Phone 001-979-845-3261, Fax 001-979-845-6028
} 
Theorem 1.2 (Armstrong and Sirakov [1]). Let $f$ be a positive continuous function on $(0, \infty)$ which satisfies

$$
\lim _{s \rightarrow \infty} e^{a s} f(s)=\infty \quad \text { for every } a>0 .
$$

Then the inequality (1.1) has no positive solution in any exterior domain of $\mathbb{R}^{2}$.

Theorem 1.2 is also sharp as explained in [1].

In this paper we study the nonexistence of positive solutions of the biharmonic inequality

$$
-\Delta^{2} v \geq f(v)
$$

in exterior domains in $\mathbb{R}^{n}$. When $n \geq 3$ we obtain the following result.

Theorem 1.3. Let $f$ be a positive continuous function on $(0, \infty)$ which satisfies

$$
\liminf _{s \rightarrow 0^{+}} \frac{f(s)}{s^{1+\frac{4}{n-2}}}>0 \quad \text { and } \quad \lim _{s \rightarrow \infty} \frac{f(s)}{s^{-1}}=\infty .
$$

Then the inequality (1.4) has no $C^{4}$ positive solution in any exterior domain of $\mathbb{R}^{n}, n \geq 3$.

Remark 1. The exponent $1+\frac{4}{n-2}$ in (1.5) is optimal because for each constant $\lambda \in\left(1+\frac{4}{n-2}, 1+\frac{4}{n-4}\right)$ (resp. $\lambda>1+\frac{4}{n-2}$ ) there exists a positive constant $C$ such that a solution of

$$
\left.-\Delta^{2} v=v^{\lambda} \quad \text { in } \quad \mathbb{R}^{n} \backslash\{0\}, n \geq 5 \text { (resp. } n=3 \text { or } 4\right)
$$

which tends to zero as $|y| \rightarrow \infty$, is $v(y)=C|y|^{\frac{-4}{\lambda-1}}$.

Remark 2. The exponent -1 in (1.5) is optimal because for each constant $\lambda<-1$, (resp. $\lambda \in$ $(-3,-1))$, there exists a positive constant $C$ such that a solution of (1.6), which tends to infinity as $|y| \rightarrow \infty$, is $v(y)=C|y|^{\frac{-4}{\lambda-1}}$.

Remark 3. We conjecture that Theorem 1.3 is true when in (1.5) the condition on $f$ at $\infty$ is replaced with

$$
\liminf _{s \rightarrow \infty} \frac{f(s)}{s^{-1}}>0
$$

It can be shown that this conjecture is true under the added assumption in Theorem 1.3 that $v$ is radial.

By Remarks 1 and 2, we see, in strong contrast to Theorem 1.1, that a growth condition on $f$ at both $s=0$ and $s=\infty$ is necessary for nonexistence of positive solutions of (1.4) in exterior domains of $\mathbb{R}^{n}, n \geq 3$.

Our two dimensional result for (1.4) is the following theorem. As is Theorem 1.2 and in contrast to Theorem 1.3, a growth condition on $f$ is only needed at $s=\infty$ for nonexistence of positive solutions of (1.4) in exterior domains of $\mathbb{R}^{2}$

Theorem 1.4. Let $f$ be a positive continuous function on $(0, \infty)$ which satisfies

$$
\liminf _{s \rightarrow \infty} \frac{f(s)}{s^{-1} \log s}>0 .
$$

Then the inequality (1.4) has no $C^{4}$ positive solution in any exterior domain of $\mathbb{R}^{2}$. 
Remark 4. The exponent -1 in (1.8) is optimal because for each constant $\lambda \in(-2,-1)$ there exists a positive constant $C$ such that a positive solution of

$$
-\Delta^{2} v \geq v^{\lambda} \quad \text { in } \quad \mathbb{R}^{2} \backslash B_{e}(0),
$$

which tends to infinity as $|y| \rightarrow \infty$, is $v(y)=2 C|y|^{2} \log |y|-|y|^{b}$ where $b=2 \lambda+4 \in(0,2)$.

Remark 5. We conjecture that Theorem 1.4 is true when (1.8) is replaced with (1.7). By Lemma 2.8 this conjecture is true under the added assumption in Theorem 1.4 that $v$ is radial.

Since there are continuous functions $f:(0, \infty) \rightarrow(0, \infty)$ satisfying (1.5) (resp. (1.8)) which are not bounded below by a convex function $g:(0, \infty) \rightarrow(0, \infty)$ satisfying (1.5) (resp. (1.8)), one cannot immediately reduce the the proof of Theorem 1.3 (resp. Theorem 1.4) to an ODE problem by the standard method of averaging which consists of replacing $f$ in (1.4) with such a $g$, averaging the resulting inequality, and using Jensen's inequality. In particular, obtaining nonexistence results for (1.4) under assumption (1.5) (resp. (1.8)) is much more difficult than obtaining them, say, for

$$
-\Delta^{2} v \geq v^{\lambda}
$$

where $\lambda \in \mathbb{R} \backslash(0,1)$ is a constant, because the function $f(v)=v^{\lambda}$ is convex. Our results when applied to (1.9) give the following corollary.

Corollary 1.1. Suppose $\lambda \in \mathbb{R}, r_{0}>0$ and $n=2$ (resp. $n \geq 3$ ). Then (1.9) has $C^{4}$ positive solutions in $\mathbb{R}^{n} \backslash B_{r_{0}}(0)$ if and only if

$$
\lambda<-1 \quad\left(\text { resp. } \lambda<-1 \quad \text { or } \quad \lambda>1+\frac{4}{n-2}\right) .
$$

Proof. The "if" part of the corollary follows by scaling, if necessary, the examples in Remarks 1, 2, and 4. The "only if" part of the corollary follows from Theorems 1.3 and 1.4 when $\lambda \neq-1$ and from Lemmas 2.8 and 2.10 when $\lambda=-1$.

The result in Corollary 1.1 above is different from the study of (1.9) in the whole space $\mathbb{R}^{n}$. Mitidieri and Pohozaev [14, Theorem 7.1, pg. 31] proved that (1.9) has no solution in $\mathbb{R}^{n}$ if $1<\lambda \leq 1+4 /(n-4)$. In particular, no entire solution exists for all $\lambda>1$ in dimensions $n=3$ and $n=4$.

Let us briefly describe the methods we employ in this paper to deal with the biharmonic inequality (1.4). The method used in [1] to prove Theorems 1.1 and 1.2 depends only on properties related to the maximum principle. Since the maximum principle does not hold for the biharmonic operator, we adopt a different approach to prove Theorems 1.3 and 1.4 which relies on a new representation formula and an a priori pointwise bound for nonnegative solutions of $-\Delta^{2} u \geq 0$ in a punctured neighborhood of the origin in $\mathbb{R}^{n}$, which we state in Appendix $\mathrm{A}$. We assume for contradiction that there exists a positive solution $v(y)$ of (1.4) in an exterior domain and apply this representation formula (A.5) and pointwise bound (A.3) to the 2-Kelvin transform $u(x)$ of the function $v(y)$. A crucial step in our approach is to show using (A.5) that the estimate (A.4) can be improved to

$$
\int_{|x|<1}-\Delta^{2} u(x) d x<\infty
$$

This will then imply that

$$
\int_{|x|<r} u(x) d x=o\left(r^{3}\right) \quad \text { as } r \rightarrow 0^{+},
$$


which will allow us to obtain with the help of Lemma 2.5 a refined representation formula for $u$, the crucial term of which is, instead of (A.6),

$$
\hat{N}(x)=\int_{B_{1}(0)} \Phi(x-y) \Delta^{2} u(y) d y .
$$

Here $\Phi$ is the fundamental solution of $\Delta^{2}$ in $\mathbb{R}^{n}$ given by

$$
\Phi(x):=A \begin{cases}|x|^{4-n} & \text { if } n \geq 5 \\ \log \frac{e}{|x|} & \text { if } n=4 \\ -|x| & \text { if } n=3 \\ -|x|^{2} \log \frac{e}{|x|} & \text { if } n=2\end{cases}
$$

where $A=A(n)$ is a positive constant. Finally we are able to raise a contradiction by providing with the help of Lemma 2.1 various estimates as $r \rightarrow 0^{+}$of expressions involving $\int_{|x|=r} \hat{N}(x) d x$.

The form and sign of the fundamental solution $\Phi$ have a large influence on the proofs of Theorems 1.3 and 1.4. The proofs in cases (1.10) and (1.11) are similar but very different from the proof in case (1.13). The proof in case (1.12) is a hybrid of the proofs in the other three cases. We have tried to avoid repetition of arguments which occur in two or more cases by giving them, without repetition, in the proofs of some lemmas in Section 2. Also, since the first few paragraphs of the proofs in cases (1.10)-(1.12) are the same, we have in Section 3 presented them only once.

For simplicity and to more easily compare our results to those in [1], we stated in Theorems 1.3 and 1.4 special cases of our more general results which are the following two theorems and which address the nonexistence of positive solutions of the inequality

$$
-\Delta^{2} v \geq|y|^{-\sigma} f(v)
$$

in exterior domains in $\mathbb{R}^{n}, n \geq 2$.

Theorem 1.5. Suppose $\sigma<2$ is a constant, $\Omega$ is a compact subset of $\mathbb{R}^{n}, n \geq 3$, and $f:(0, \infty) \rightarrow$ $(0, \infty)$ is a continuous function satisfying

$$
\liminf _{s \rightarrow 0^{+}} \frac{f(s)}{s^{1+\frac{4-\sigma}{n-2}}}>0 \quad \text { and } \quad \lim _{s \rightarrow \infty} \frac{f(s)}{s^{-1+\frac{\sigma}{2}}}=\infty .
$$

Then there does not exist a $C^{4}$ positive solution $v(y)$ of (1.14) in $\mathbb{R}^{n} \backslash \Omega$.

Theorem 1.6. Suppose $\sigma \in[0,2)$ is a constant, $\Omega$ is a compact subset of $\mathbb{R}^{2}$, and $f:(0, \infty) \rightarrow$ $(0, \infty)$ is a continuous function satisfying

$$
\liminf _{s \rightarrow \infty} \frac{f(s)}{s^{-1+\frac{\sigma}{2}}} \frac{\prod_{i=2}^{k} \log ^{i} s}{(\log s)^{1-\frac{\sigma}{2}}}>0
$$

for some integer $k \geq 2$ where $\log ^{2}=\log \circ \log , \log ^{3}=\log \circ \log \circ \log$, etc. Then there does not exist a $C^{4}$ positive solution $v(y)$ of (1.14) in $\mathbb{R}^{2} \backslash \Omega$.

Remark 6. Theorems 1.5 and 1.6 with $\sigma=0$ immediately imply Theorems 1.3 and 1.4, respectively. 
Remark 7. Similar to Remarks 1, 2, and 4, the exponents $1+\frac{4-\sigma}{n-2}$ and $-1+\frac{\sigma}{2}$ in (1.15) are optimal and so is the exponent $-1+\frac{\sigma}{2}$ in (1.16).

Mitidieri and Pohozaev [14, Remark 9.1] have shown that the problem

$$
\pm \Delta^{m} u \geq|x|^{-2 m}|u|^{q}, \quad x \in \mathbb{R}^{n} \backslash\{0\},
$$

has no nontrivial weak solution if $m, n \geq 1$ and $q>1$. Also, nonnegative solutions of problems of the form

$$
-\Delta^{m} u=f(x, u) \quad \text { or } \quad-\Delta^{m} u \geq f(x, u)
$$

when $f$ is a nonnegative function have been studied in [4, 5, 10, 11, 12, 13, 15, 16, 17] and elsewhere. These problems arise naturally in conformal geometry and in the study of the Sobolev embedding of $H^{2 m}$ into $L^{\frac{2 n}{n-2 m}}$.

Nonexistence results for entire solutions $u$ of problems (1.17) can be used to obtain, via scaling methods, estimates of solutions of boundary value problems associated with (1.17). An excellent reference for polyharmonic boundary value problems is [8].

Also, weak solutions of $\Delta^{m} u=\mu$, where $\mu$ is a measure on a subset of $\mathbb{R}^{n}$, have been studied in [3, 6, 7], and removable isolated singularities of $\Delta^{m} u=0$ have been studied in [12].

\section{Preliminary results}

In this section we provide some results needed for the proofs of Theorems 1.5 and 1.6 .

Lemma 2.1. Suppose $m \geq 1$ and $n \geq 2$ are integers and $\Gamma(z)=\Gamma(|z|)$ is a radial solution of $\Delta^{m} \Gamma=0$ in $\mathbb{R}^{n} \backslash\{0\}$. For each $r>0$, let

$$
u(x ; r)=\frac{1}{\left|\partial B_{r}\right|} \int_{|y|=r} \Gamma(|x-y|) d S_{y} \quad \text { for } x \in \mathbb{R}^{n} .
$$

Then

$$
u(x ; r)= \begin{cases}\sum_{i=0}^{m-1} \frac{\Delta^{i} \Gamma(r)}{\alpha_{i}}|x|^{2 i}, & \text { if }|x|<r \\ \sum_{i=0}^{m-1} \frac{\Delta^{i} \Gamma(|x|)}{\alpha_{i}} r^{2 i}, & \text { if }|x|>r\end{cases}
$$

where $\alpha_{0}=1$ and

$$
\alpha_{i}=\Delta^{i}|x|^{2 i}=i ! 2^{i}[n(n+2)(n+4) \cdots(n+2 i-2)] \quad \text { for } i=1,2, \ldots, m-1 .
$$

Proof. Since $u(x ; r)$ is radial in $x$, we can define $v:[0, \infty) \times(0, \infty) \rightarrow \mathbb{R}$ by $v(|x|, r)=u(x ; r)$ and to prove Lemma 2.1 it suffices to prove

$$
v(\rho, r)= \begin{cases}\sum_{i=0}^{m-1} \frac{\Delta^{i} \Gamma(r)}{\alpha_{i}} \rho^{2 i}, & \text { if } \rho<r \\ \sum_{i=0}^{m-1} \frac{\Delta^{i} \Gamma(\rho)}{\alpha_{i}} r^{2 i}, & \text { if } \rho>r .\end{cases}
$$

Since

$$
\begin{aligned}
v(\rho, r) & =\frac{1}{\left|\partial B_{\rho}\right|} \int_{|x|=\rho} v(|x|, r) d S_{x} \\
& =\frac{1}{\left|\partial B_{\rho}\right|} \frac{1}{\left|\partial B_{r}\right|} \int_{|x|=\rho} \int_{|y|=r} \Gamma(|x-y|) d S_{y} d S_{x}
\end{aligned}
$$


we see that

$$
v(\rho, r)=v(r, \rho) \quad \text { for }(\rho, r) \in(0, \infty) \times(0, \infty) .
$$

Since $u(x ; r)$ is a $C^{\infty}$ radial solution of $\Delta^{m} u=0$ in $B_{r}(x)$ there are constants $c_{i}$ such that

$$
u(x ; r)=\sum_{i=0}^{m-1} c_{i}|x|^{2 i} \quad \text { for }|x|<r .
$$

Hence $\left(\Delta^{j} u\right)(0 ; r)=c_{j} \Delta^{j}|x|^{2 j}=c_{j} \alpha_{j}$ for $j=0,1, \ldots, m-1$. On the other hand, it follows from (2.1) that $\left(\Delta^{j} u\right)(0 ; r)=\Delta^{j} \Gamma(r)$ and hence

$$
c_{j}=\frac{\Delta^{j} \Gamma(r)}{\alpha_{j}} \quad \text { for } j=0,1,2, \ldots, m-1 .
$$

Thus (2.2), and hence (2.3), holds for $|x|=\rho<r$ and by (2.4) we have (2.3) also holds for $\rho>r$.

Lemma 2.2. Suppose $r \in\left(0, \frac{1}{4}\right]$ and $\alpha \geq 1$. Then

$$
\left(\log \frac{e|y|}{r}\right)^{\alpha}-|y|^{2} \geq \frac{1}{4}\left(\log \frac{e|y|}{r}\right)^{\alpha} \quad \text { for } \quad r \leq|y| \leq 1 .
$$

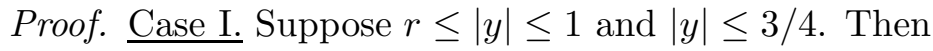

$$
\frac{3}{4}\left(\log \frac{e|y|}{r}\right)^{\alpha} \geq \frac{3}{4}(\log e)^{\alpha} \geq|y|^{2} .
$$

Case II. Suppose $r \leq|y| \leq 1$ and $|y| \geq 3 / 4$. Then

$$
\frac{3}{4}\left(\log \frac{e|y|}{r}\right)^{\alpha} \geq \frac{3}{4}(\log 3 e)^{\alpha} \geq \frac{3}{4} 2^{\alpha} \geq|y|^{2} .
$$

Lemma 2.3. Suppose $f:(0, \infty) \rightarrow(0, \infty)$ is a continuous function satisfying

$$
\lim _{s \rightarrow \infty} s^{\alpha} f(s)=\infty \quad \text { for some constant } \alpha>0 .
$$

Then there exists a continuous function $\hat{f}:(0, \infty) \rightarrow(0, \infty)$ such that $\hat{f} \leq f$ on $(0, \infty), \hat{f}=f$ on $(0,1], \hat{f}$ is decreasing on $[1, \infty)$, and

$$
\lim _{s \rightarrow \infty} s^{\alpha} \hat{f}(s)=\infty
$$

Proof. Define $\hat{f}:(0, \infty) \rightarrow(0, \infty)$ by

$$
\hat{f}(s)= \begin{cases}f(s), & \text { if } 0<s \leq 1 \\ \min _{1 \leq \zeta \leq s} f(\zeta), & \text { if } 1 \leq s<\infty .\end{cases}
$$

Clearly $\hat{f}$ is continuous, $\hat{f} \leq f$, and $\hat{f}$ is decreasing on $[1, \infty)$. Let $M>1$. Choose $s_{0}>1$ such that $\zeta^{\alpha} f(\zeta) \geq M$ for $\zeta \geq s_{0}$. Choose $s_{1}>s_{0}$ such that $s_{1}^{\alpha} \hat{f}\left(s_{0}\right) \geq M$. Then for $s \geq s_{1}$ we have

$$
\begin{aligned}
s^{\alpha} \hat{f}(s) & =s^{\alpha} \min \left\{\hat{f}\left(s_{0}\right), \min _{s_{0} \leq \zeta \leq s} f(\zeta)\right\} \\
& \geq \min \left\{s_{1}^{\alpha} \hat{f}\left(s_{0}\right), \min _{s_{0} \leq \zeta \leq s} \zeta^{\alpha} f(\zeta)\right\} \geq M
\end{aligned}
$$

which proves (2.5). 
Lemma 2.4. Suppose $h$ is a solution of

$$
\Delta^{2} h=0 \quad \text { in } \quad \overline{B_{1}(0)} \backslash\{0\} \subset \mathbb{R}^{n}, n \geq 3 .
$$

Then there exist constants $c_{i}, i=1, \ldots, 5$, such that for $0<r<1$ we have

$$
\int_{r<|x|<1}|x|^{-4} h(x) d x= \begin{cases}c_{1} r^{n-2}+c_{2} r^{n-4}+c_{3} \log r+c_{4} r^{-2}+c_{5} & \text { if } n=3 \text { or } n \geq 5 \\ c_{1} r^{2}+c_{2} \log r+c_{3}(\log r)^{2}+c_{4} r^{-2}+c_{5} & \text { if } n=4 .\end{cases}
$$

Proof. It follows from (2.6) that there exist constants $\hat{c}_{i}, i=1,2,3,4$, such that for $0<\rho<1$ we have

$$
\bar{h}(\rho):=\frac{1}{\left|\partial B_{1}\right| \rho^{n-1}} \int_{|x|=\rho} h(x) d S_{x}= \begin{cases}\hat{c}_{1} \rho^{2}+\hat{c}_{2}+\hat{c}_{3} \rho^{4-n}+\hat{c}_{4} \rho^{2-n} & \text { if } n=3 \text { or } n \geq 5 \\ \hat{c}_{1} \rho^{2}+\hat{c}_{2}+\hat{c}_{3} \log \rho+\hat{c}_{4} \rho^{-2} & \text { if } n=4 .\end{cases}
$$

Thus

$$
\begin{aligned}
\int_{r<|x|<1}|x|^{-4} h(x) d x & =\int_{r}^{1} \rho^{-4}\left(\int_{|x|=\rho} h(x) d S_{x}\right) d \rho=\left|\partial B_{1}\right| \int_{r}^{1} \rho^{n-5} \bar{h}(\rho) d \rho \\
& = \begin{cases}\left|\partial B_{1}\right| \int_{r}^{1}\left(\hat{c}_{1} \rho^{n-3}+\hat{c}_{2} \rho^{n-5}+\hat{c}_{3} \rho^{-1}+\hat{c}_{4} \rho^{-3}\right) d \rho & \text { if } n=3 \text { or } n \geq 5 \\
\left|\partial B_{1}\right| \int_{r}^{1}\left(\hat{c}_{1} \rho+\hat{c}_{2} \rho^{-1}+\hat{c}_{3} \rho^{-1} \log \rho+\hat{c}_{4} \rho^{-3}\right) d \rho & \text { if } n=4\end{cases}
\end{aligned}
$$

from which we obtain Lemma 2.4 .

Lemma 2.5. Suppose $v \in L_{\text {loc }}^{1}(B)$, where $B=B_{1}(0) \subset \mathbb{R}^{n}, n \geq 2$. If

$$
\Delta^{2} v=0 \quad \text { in } \mathcal{D}^{\prime}(B \backslash\{0\})
$$

and

$$
\int_{|x|<r}|v(x)| d x=o\left(r^{3}\right) \quad \text { as } \quad r \rightarrow 0^{+}
$$

then for some constant $a$ and some $C^{\infty}$ solution $H$ of $\Delta^{2} H=0$ in $B$ we have

$$
v=a \Phi+H \quad \text { in } \quad B \backslash\{0\}
$$

where $\Phi$ is given by (1.10)-(1.13).

Proof. Since the support of $\Delta^{2} v$ is a single point we have $\Delta^{2} v$ is a finite linear combination of the delta function and its derivatives:

$$
\Delta^{2} v=\sum_{|\beta| \leq k} a_{\beta} D^{\beta} \delta \quad \text { in } \quad \mathcal{D}^{\prime}(B) .
$$

We now use a method of Brezis and Lions [2] to show $a_{\beta}=0$ for $|\beta| \geq 1$. Choose $\varphi \in C_{0}^{\infty}(B)$ such that $(-1)^{|\beta|}\left(D^{\beta} \varphi\right)(0)=a_{\beta}$ for $|\beta| \leq k$. Let $\varphi_{\varepsilon}(x)=\varphi\left(\frac{x}{\varepsilon}\right)$. Then, for $0<\varepsilon<1, \varphi_{\varepsilon} \in C_{0}^{\infty}(B)$, and

$$
\begin{aligned}
\int v \Delta^{2} \varphi_{\varepsilon} & =\left(\Delta^{2} v\right)\left(\varphi_{\varepsilon}\right)=\sum_{|\beta| \leq k} a_{\beta}\left(D^{\beta} \delta\right) \varphi_{\varepsilon} \\
& =\sum_{|\beta| \leq k} a_{\beta}(-1)^{|\beta|} \delta\left(D^{\beta} \varphi_{\varepsilon}\right)=\sum_{|\beta| \leq k} a_{\beta}(-1)^{|\beta|}\left(D^{\beta} \varphi_{\varepsilon}\right)(0) \\
& =\sum_{|\beta| \leq k} a_{\beta}(-1)^{|\beta|} \frac{1}{\varepsilon^{|\beta|}}\left(D^{\beta} \varphi\right)(0)=\sum_{|\beta| \leq k} a_{\beta}^{2} \frac{1}{\varepsilon^{|\beta|}} .
\end{aligned}
$$


On the other hand,

$$
\begin{aligned}
\int v \Delta^{2} \varphi_{\varepsilon} & =\int v(x) \frac{1}{\varepsilon^{4}} \Delta^{2} \varphi\left(\frac{x}{\varepsilon}\right) d x \\
& \leq \frac{C}{\varepsilon^{4}} \int_{|x|<\varepsilon}|v(x)| d x=o\left(\varepsilon^{-1}\right) \quad \text { as } \quad \varepsilon \rightarrow 0^{+}
\end{aligned}
$$

by (2.7). Hence $a_{\beta}=0$ for $|\beta| \geq 1$ and consequently, letting $a=a_{0}$, we have $\Delta^{2}(v-a \Phi)=0$ in $\mathcal{D}^{\prime}(B)$. Thus the lemma follows from the fact that weakly biharmonic functions are $C^{\infty}$.

Lemma 2.6. Suppose $u(x)$ is a $C^{4}$ positive solution of

$$
-\Delta^{2} u \geq \alpha|x|^{\frac{2 \sigma-4 n}{n-2}} u(x)^{\lambda} \quad \text { in } \quad \overline{B_{1}(0)} \backslash\{0\} \subset \mathbb{R}^{n}, n \geq 3,
$$

where $\alpha>0$ and $\sigma<4$ are constants and $\lambda=1+\frac{4-\sigma}{n-2}$. Then

$$
\liminf _{r \rightarrow 0^{+}} \frac{\bar{u}(r)}{r^{2} J(r)}=0
$$

where $\bar{u}(r)$ is the average of $u$ on the sphere $|x|=r$ and

$$
J(r):=\int_{r<|y|<1}|y|^{2-n}\left(-\Delta^{2} u(y)\right) d y \quad \text { for } \quad 0<r<1 .
$$

Proof. Suppose for contradiction that there exists $\varepsilon, r_{0} \in(0,1)$ such that $\bar{u}(r) \geq \varepsilon r^{2} J(r)$ for $0<r \leq r_{0}$. Then, letting $C$ denote a positive constant whose value may change from line to line, we have for $0<r \leq r_{0}$ that

$$
\begin{aligned}
-J^{\prime}(r) & =\int_{|y|=r}|y|^{2-n}\left(-\Delta^{2} u(y)\right) d S_{y} \\
& =r^{2-n}\left|\partial B_{1}\right| r^{n-1}\left(-\overline{\Delta^{2} u}(r)\right) \\
& =C r\left(-\overline{\Delta^{2} u}(r)\right) \\
& \geq C r\left(r^{\frac{2 \sigma-4 n}{n-2}}(\bar{u}(r))^{\lambda}\right) \\
& \geq C r^{1+\frac{2 \sigma-4 n}{n-2}}\left(\varepsilon r^{2} J(r)\right)^{\lambda} \\
& =C r^{1+\frac{2 \sigma-4 n}{n-2}+2 \lambda} J(r)^{\lambda} \\
& =C r^{-1} J(r)^{\lambda} .
\end{aligned}
$$

Consequently $-J^{\prime}(r) J(r)^{-\lambda} \geq C r^{-1}$ for $0<r \leq r_{0}$ which implies

$$
\frac{1}{(\lambda-1) J\left(r_{0}\right)^{\lambda-1}} \geq \frac{1}{\lambda-1}\left[\frac{1}{J\left(r_{0}\right)^{\lambda-1}}-\frac{1}{J(r)^{\lambda-1}}\right] \geq C \log \frac{r_{0}}{r} \rightarrow \infty
$$

as $r \rightarrow 0^{+}$, a contradiction, which proves the lemma.

Lemma 2.7. Suppose $u$ is a $C^{4}$ positive solution of

$$
-\Delta^{2} u>0 \quad \text { in } \quad B_{2}(0) \backslash\{0\} \subset \mathbb{R}^{n}, \quad n \geq 2,
$$


such that

$$
\int_{|x|<1} \varphi(x) u(x) d x<\infty
$$

where $\varphi: \overline{B_{1}(0)} \backslash\{0\} \rightarrow(0, \infty)$ is a continuous radial function satisfying

$$
\int_{|x|<1} \varphi(x) d x=\infty
$$

Then

$$
\int_{|x|<1}-\Delta^{2} u(x) d x<\infty
$$

Proof. Let $F(\rho)=-\overline{\Delta^{2} u}(\rho)=-\Delta^{2} \bar{u}(\rho)$. Then for some constants $c_{1}$ and $c_{2}$ we have for $0<r \leq 1$ that

$$
\Delta \bar{u}(r)= \begin{cases}c_{1}+c_{2} \log \frac{1}{r}-(N F)(r) & \text { if } n=2 \\ c_{1}+c_{2} r^{2-n}-(N F)(r)<0 & \text { if } n \geq 3\end{cases}
$$

where $(N F)(r)=\int_{r}^{1} s^{-(n-1)} \int_{s}^{1} \rho^{n-1} F(\rho) d \rho d s$.

Suppose for contradiction that (2.12) is false. Then $\int_{0}^{1} \rho^{n-1} F(\rho) d \rho=\infty$ and hence as $r \rightarrow 0^{+}$ we have

$$
(N F)(r)>> \begin{cases}\log \frac{1}{r} & \text { if } n=2 \\ r^{2-n} & \text { if } n \geq 3 .\end{cases}
$$

Thus for small $r>0$ we have $\Delta \bar{u}(r)<0$. Hence the positivity of $\bar{u}$ implies $\bar{u}>\varepsilon>0$ for small $r>0$, which together with (2.10) and (2.11) gives a contradiction and completes the proof of Lemma 2.7.

Lemma 2.8. There does not exist a positive radial solution of

$$
-\Delta^{2} v \geq|y|^{-\sigma} f(v) \quad \text { in } \quad \mathbb{R}^{2} \backslash B_{r_{0 / 2}}(0)
$$

where $r_{0} \geq 2$ and $\sigma \in[0,2)$ are constants and $f:(0, \infty) \rightarrow(0, \infty)$ is a continuous function such that

$$
\liminf _{s \rightarrow \infty} \frac{f(s)}{s^{-1+\frac{\sigma}{2}}}>0
$$

Proof. Suppose for contradiction that $v(r)$ is a positive radial solution of (2.13). Let $F(r)=$ $-\left(\Delta^{2} v\right)(r)$ and $(N F)(r)=\int_{r_{0}}^{r} \frac{1}{s} \int_{r_{0}}^{s} \rho F(\rho) d \rho d s$. Then for some constants $c_{1}, \ldots, c_{4}$ we have

$$
v(r)=c_{1}+c_{2} \log r+c_{3} r^{2}+c_{4} r^{2} \log r-\left(N^{2} F\right)(r) .
$$

If $\int_{r_{0}}^{s} \rho F(\rho) d \rho \rightarrow \infty$ as $s \rightarrow \infty$ then $(N F)(r)>>\log r$ as $r \rightarrow \infty$ and hence $\left(N^{2} F\right)(r)>>r^{2} \log r$ as $r \rightarrow \infty$ which together with (2.15) contradicts the positivity of $v$. Thus

$$
\int_{r_{0}}^{\infty} \rho F(\rho) d \rho<\infty
$$

We claim that

$$
\liminf _{r \rightarrow \infty} v(r)=0
$$


To see this, suppose for contradiction that (2.17) is false. Then for some $\varepsilon>0$ we have $v(r)>\varepsilon$ for $r_{0} \leq r<\infty$. Thus by (2.13), (2.14) and (2.15) we have

$$
\begin{aligned}
F(r) & =-\Delta^{2} v(r) \geq r^{-\sigma} f(v(r)) \geq \frac{1}{C r^{\sigma} v(r)^{1-\frac{\sigma}{2}}} \\
& \geq \frac{1}{C r^{\sigma}\left(r^{2} \log r\right)^{1-\frac{\sigma}{2}}} \geq \frac{1}{C r^{2} \log r} \quad \text { for } r \text { large }
\end{aligned}
$$

which contradicts (2.16) and proves (2.17).

By (2.16),

$$
(\hat{N} F)(r):=\int_{r_{0}}^{r} \frac{1}{s} \int_{s}^{\infty} \rho F(\rho) d \rho d s=o(\log r) \quad \text { as } \quad r \rightarrow \infty
$$

and thus

$$
(N \hat{N} F)(r)=o\left(r^{2} \log r\right) \quad \text { as } \quad r \rightarrow \infty .
$$

Since $v(r)$ solves (2.13), there exist constants $\hat{c_{1}}, \ldots, \hat{c_{4}}$ such that

$$
v(r)=\hat{c}_{1}+\hat{c}_{2} \log r+\hat{c}_{3} r^{2}+\hat{c}_{4} r^{2} \log r+(N \hat{N} F)(r) .
$$

Since $v>0$, it follows from (2.18) and (2.19) that

$$
\hat{c}_{4} \geq 0
$$

If $(\hat{N} F)(r) \rightarrow \infty$ then $(N \hat{N} F)(r)>>r^{2}$ as $r \rightarrow \infty$ which together with (2.19) and (2.20) implies $v(r) \rightarrow \infty$ as $r \rightarrow \infty$ which contradicts (2.17). Hence $(\hat{N} F)(r)$ is bounded. Thus

$$
(\hat{\hat{N}} F)(r):=\int_{r}^{\infty} \frac{1}{s} \int_{s}^{\infty} \rho F(\rho) d \rho d s=o(1) \quad \text { as } r \rightarrow \infty
$$

which implies

$$
(N \hat{\hat{N}} F)(r)=o\left(r^{2}\right) \quad \text { as } \quad r \rightarrow \infty .
$$

Since $v$ solves (2.13) there exist constants $\hat{\hat{c}}_{1}, \ldots, \hat{\hat{c}}_{4}$ such that

$$
v(r)=\hat{\hat{c}}_{1}+\hat{\hat{c}}_{2} \log r+\hat{\hat{c}}_{3} r^{2}+\hat{\hat{c}}_{4} r^{2} \log r-(N \hat{\hat{N}} F)(r) .
$$

By (2.21) and (2.22) and the positivity of $v$ we have $\hat{\hat{c}}_{4} \geq 0$ and then by (2.17), $\hat{\hat{c}}_{4}=0$. Hence by (2.21) and (2.22) and the positivity of $v$ we have $\hat{\hat{c}}_{3} \geq 0$ and then by (2.17), $\hat{\hat{c}}_{3}=0$. Thus by (2.22)

$$
v(r)=\hat{\hat{c}}_{1}+\hat{\hat{c}}_{2} \log r-(N \hat{\hat{N}} F)(r)
$$

and so $-\Delta v=\hat{\hat{N}} F>0$ which together with the positivity of $v$ contradicts (2.17) and completes the proof of Lemma 2.8,

Lemma 2.9. Suppose $x, y \in \mathbb{R}^{2}$ and $y \neq 0$. Then

$$
I(x, y):=\int_{0}^{1}(1-t) \log \frac{|y|}{|y-t x|} d t \leq 2 \int_{0}^{1} \log \frac{1}{s} d s<\infty .
$$


Proof. Since $I(0, y)=0$ we can assume $x \neq 0$. Under the change of variables $\tau=\frac{|x|}{|y|} t$ we have

$$
\begin{aligned}
I(x, y) & =\frac{|y|}{|x|} \int_{0}^{\frac{|x|}{|y|}}\left(1-\frac{|y|}{|x|} \tau\right) \log \frac{1}{\left|\frac{y}{|y|}-\tau \frac{x}{|x|}\right|} d \tau \\
& \leq \frac{|y|}{|x|} \int_{0}^{\frac{|x|}{|y|}}\left(1-\frac{|y|}{|x|} \tau\right) \log \frac{1}{|1-\tau|} d \tau \\
& =\varphi\left(\frac{|x|}{|y|}\right)
\end{aligned}
$$

where $\varphi:(0, \infty) \rightarrow \mathbb{R}$ is given by

$$
\begin{aligned}
& \varphi(\rho):=\frac{1}{\rho} \int_{0}^{\rho}\left(1-\frac{\tau}{\rho}\right) \log \frac{1}{|1-\tau|} d \tau \\
& \leq \frac{1}{\rho} \int_{0}^{\min \{\rho, 2\}} \log \frac{1}{|1-\tau|} d \tau \\
& \leq \begin{cases}\frac{1}{2} \int_{0}^{2} \log \frac{1}{|1-\tau|} d \tau, & \text { if } \rho \geq 2 \\
\frac{1}{\rho} \int_{0}^{\rho} \log \frac{1}{|1-\tau|} d \tau, & \text { if } 0<\rho \leq 2\end{cases} \\
& \leq \int_{0}^{2} \log \frac{1}{|1-\tau|} d \tau=2 \int_{0}^{1} \log \frac{1}{s} d s \text {. }
\end{aligned}
$$

Lemma 2.10. There does not exist a $C^{4}$ positive solution of

$$
-\Delta^{2} v \geq v^{-1} \quad \text { in } \mathbb{R}^{n} \backslash \overline{B_{R / 2}(0)}, n \geq 3,
$$

where $R$ is a positive constant.

Proof. By averaging (2.23) we can assume $v$ is radial. Let $F(r)=-\Delta^{2} v(r)$. Then

$$
v(r)=c_{1}+c_{2} r^{2}+c_{3} r^{2-n}+c_{4} \Phi(r)-\left(N^{2} F\right)(r) \quad \text { for } r \geq R
$$

where $\Phi(r)$ is given by (1.10) $-(1.12)$ and

$$
(N F)(r):=\int_{R}^{r} s^{1-n} \int_{R}^{s} \rho^{n-1} F(\rho) d \rho d s \geq 0 .
$$

Thus for some positive constant $C$ we have $v(r)<C r^{2}$ for $r \geq R$, which implies

$$
F(r)=-\Delta^{2} v(r) \geq v(r)^{-1} \geq \frac{1}{C r^{2}} \quad \text { for } r \geq R
$$

Hence $(N F)(r) \rightarrow \infty$ as $r \rightarrow \infty$. Thus $\left(N^{2} F\right)(r)>>r^{2}$ as $r \rightarrow \infty$ which together with (2.24) contradicts the positivity of $v(r)$. 


\section{Beginning of the Proof of Theorem 1.5}

In this section we begin the proof of Theorem 1.5. In Sections 4, 5, and 6, we will complete the proof of Theorem 1.5 when $n \geq 5, n=4$, and $n=3$, respectively.

Beginning of the proof of Theorem 1.5. Suppose for contradiction that $v(y)$ is a $C^{4}$ positive solution of (1.14) in $\mathbb{R}^{n} \backslash \Omega$. By scaling $v$, we can assume $\Omega=\overline{B_{1 / 2}(0)}$ and

$$
f(s) \geq s^{1+\frac{4-\sigma}{n-2}} \quad \text { for } \quad 0<s \leq 1 .
$$

Moreover, by Lemma 2.3, we can assume

$$
f \text { is decreasing on }[1, \infty) \text {. }
$$

Let $u(x)=|y|^{n-4} v(y), y=\frac{x}{|x|^{2}}$ be the 2-Kelvin transform of $v(y)$. Then

$$
v(y)=|x|^{n-4} u(x) \quad \text { and } \quad \Delta^{2} v(y)=|x|^{n+4} \Delta^{2} u(x) .
$$

(See [16] and [17].) It follows therefore from (1.14) and (3.1) that $u(x)$ is a $C^{4}$ positive solution of

$$
-\Delta^{2} u(x) \geq\left\{\begin{array}{ll}
|x|^{\frac{2 \sigma-4 n}{n-2}} u(x)^{1+\frac{4-\sigma}{n-2}}, & \text { if } 0<u(x) \leq|x|^{4-n} \\
|x|^{-n-4+\sigma} f\left(|x|^{n-4} u(x)\right), & \text { if } u(x) \geq|x|^{4-n}
\end{array} \quad \text { in } B_{2}(0) \backslash\{0\} .\right.
$$

Let $\Psi$ and $N$ be as defined in Appendix $\mathrm{A}$, Since $u$ is a $C^{4}$ positive solution of (A.2), it follows from Theorem A.1 that $u$ satisfies (A.3), (A.4), and (A.5).

By (A.5) and Lemma 2.4 there exist constants $c_{i}, i=1, \ldots, 5$, such that for $0<r<1$ we have

$$
\begin{aligned}
\int_{r<|x|<1}|x|^{-4} u(x) d x= & \int_{r<|x|<1}|x|^{-4} N(x) d x \\
& + \begin{cases}c_{1} r^{n-2}+c_{2} r^{n-4}+c_{3} \log \frac{1}{r}+c_{4} r^{-2}+c_{5} & \text { if } n=3 \text { or } n \geq 5 \\
c_{1} r^{2}+c_{2} \log \frac{e}{r}+c_{3}\left(\log \frac{e}{r}\right)^{2}+c_{4} r^{-2}+c_{5} & \text { if } n=4 .\end{cases}
\end{aligned}
$$

\section{Completion of the Proof of Theorem 1.5 when $n \geq 5$}

When $n \geq 5$, we complete in this section the proof of Theorem 1.5 which we began in Section 3 .

Completion of the proof of Theorem 1.5 when $n \geq 5$. For $x \in \mathbb{R}^{n}, n \geq 5$, we see by Lemma 2.1 that

$$
\frac{1}{\left|\partial B_{r}\right|} \int_{|y|=r} \frac{1}{|x-y|^{n-4}} d S_{y}= \begin{cases}r^{4-n}-\frac{n-4}{n} r^{2-n}|x|^{2}, & \text { if }|x|<r \\ |x|^{4-n}-\frac{n-4}{n} r^{2}|x|^{2-n}, & \text { if }|x|>r .\end{cases}
$$

It therefore follows from equations (1.10) and (A.1) that for $r>0$ we have

$$
\frac{1}{A\left|\partial B_{r}\right|} \int_{|x|=r} \Psi(x, y) d S_{x}= \begin{cases}-\frac{n-4}{n} r^{2-n}|y|^{2}, & \text { if }|y|<r \\ -r^{4-n} p\left(\frac{r}{|y|}\right), & \text { if }|y| \geq r\end{cases}
$$

where $p(t):=1-t^{n-4}+\frac{n-4}{n} t^{n-2}$ is bounded between positive constants for $0 \leq t \leq 1$. Hence

$$
-\int_{|x|=r} \Psi(x, y) d S_{x} \sim\left\{\begin{array}{ll}
2 r|y|^{2}, & \text { if }|y|<r \\
r^{3}, & \text { if }|y| \geq r
\end{array} \quad \text { for } \quad(r, y) \in(0, \infty) \times \mathbb{R}^{n} .\right.
$$


(If $f$ and $g$ are nonnegative functions defined on a set $S$ then when we write " $f(X) \sim g(X)$ for $X \in S^{\prime \prime}$ we mean there exist positive constants $C_{1}$ and $C_{2}$ such that $C_{1} g(X) \leq f(X) \leq C_{2} g(X)$ for all $X \in S$.) Thus by (A.6), for $0<r \leq \frac{1}{4}$, we have

$$
\begin{aligned}
\int_{r<|x|<1}|x|^{-4} N(x) d x & =\int_{|y|<1} \int_{r}^{1} \rho^{-4} \int_{|x|=\rho}-\Psi(x, y) d S_{x} d \rho\left(-\Delta^{2} u(y)\right) d y \\
& \sim \int_{r<|y|<1}\left(\int_{|y|}^{1} 2 \rho^{-3}|y|^{2} d \rho+\int_{r}^{|y|} \rho^{-1} d \rho\right)\left(-\Delta^{2} u(y)\right) d y \\
& +\int_{|y|<r}\left(\int_{r}^{1} 2 \rho^{-3}|y|^{2} d \rho\right)\left(-\Delta^{2} u(y)\right) d y \\
& \sim \int_{r<|y|<1}\left(\log \frac{e|y|}{r}\right)\left(-\Delta^{2} u(y)\right) d y+g(r)
\end{aligned}
$$

by Lemma 2.2 with $\alpha=1$ where

$$
0<g(r):=\left(\frac{1}{r^{2}}-1\right) \int_{|y|<r}|y|^{2}\left(-\Delta^{2} u(y)\right) d y=o\left(\frac{1}{r^{2}}\right) \quad \text { as } \quad r \rightarrow 0^{+}
$$

by (A.4).

Let $\varphi(t, r)=t^{-2} \log \frac{e t}{r}$. Since $\varphi_{t}(t, r)<0$ for $t \geq r>0$, we see for $0<r<1$ that

$$
\begin{aligned}
\int_{r<|y|<1}\left(\log \frac{e|y|}{r}\right) & \left(-\Delta^{2} u(y)\right) d y=\int_{r<|y|<1} \varphi(|y|, r)|y|^{2}\left(-\Delta^{2} u(y)\right) d y \\
& \leq \varphi(r, r) \int_{r<|y|<\sqrt{r}}|y|^{2}\left(-\Delta^{2} u(y)\right) d y+\varphi(\sqrt{r}, r) \int_{\sqrt{r}<|y|<1}|y|^{2}\left(-\Delta^{2} u(y)\right) d y \\
& \leq \frac{1}{r^{2}} \int_{r<|y|<\sqrt{r}}|y|^{2}\left(-\Delta^{2} u(y)\right) d y+\frac{1}{r}\left(\log \frac{e}{\sqrt{r}}\right) \int_{|y|<1}|y|^{2}\left(-\Delta^{2} u(y)\right) d y \\
& =o\left(r^{-2}\right) \text { as } r \rightarrow 0^{+}
\end{aligned}
$$

by (A.4). It follows therefore from (4.2) and (4.3) that

$$
\int_{r<|x|<1}|x|^{-4} N(x) d x=o\left(r^{-2}\right) \quad \text { as } \quad r \rightarrow 0^{+} .
$$

Hence, by (3.4) and the positivity of $u$ we see that the constant $c_{4}$ in (3.4) is nonnegative and thus by (3.4), (4.2), and the positivity of $g$ we have

$$
\int_{r<|x|<1}|x|^{-4} u(x) d x \geq \frac{1}{C} \int_{r<|y|<1}\left(\log \frac{e|y|}{r}\right)\left(-\Delta^{2} u(y)\right) d y-C \log \frac{e}{r} \quad \text { for } \quad 0<r<\frac{1}{4}
$$

where $C$ is a positive constant independent of $r$.

By (A.3) there exists a constant $M>1$ such that $0<u(x) \leq M|x|^{2-n}$ for $0<|x| \leq 1$. Define $I_{1}, I_{2}:(0,1) \rightarrow[0, \infty)$ by

$$
I_{1}(r):=M \int_{x \in S_{1}(r)}|x|^{-n-2} d x \quad \text { and } \quad I_{2}(r):=\int_{x \in S_{2}(r)}|x|^{-4} u(x) d x
$$

where

$$
S_{1}(r):=\left\{x \in \mathbb{R}^{n}: r<|x|<1 \text { and }|x|^{4-n}<u(x) \leq M|x|^{2-n}\right\}
$$


and

$$
S_{2}(r):=\left\{x \in \mathbb{R}^{n}: r<|x|<1 \text { and } 0<u(x) \leq|x|^{4-n}\right\} .
$$

Then $S_{1}(r) \cup S_{2}(r)=B_{1}(0)-\overline{B_{r}(0)}$,

$$
I_{2}(r)=O\left(\log \frac{1}{r}\right) \quad \text { as } \quad r \rightarrow 0^{+},
$$

and for $0<r<\frac{1}{4}$ we have

$$
\begin{aligned}
I_{1}(r)+I_{2}(r) & \geq \int_{r<|x|<1}|x|^{-4} u(x) d x \\
& \geq \frac{1}{C} \int_{r<|y|<1}\left(\log \frac{e|y|}{r}\right)\left(-\Delta^{2} u(y)\right) d y-C \log \frac{e}{r}
\end{aligned}
$$

by (4.4).

By (3.3) we have

$$
\int_{r<|y|<1}\left(\log \frac{e|y|}{r}\right)\left(-\Delta^{2} u(y)\right) d y \geq J_{1}(r)+J_{2}(r) \quad \text { for } \quad 0<r<1
$$

where

$$
J_{2}(r):=\int_{S_{2}(r)}|y|^{\frac{2 \sigma-4 n}{n-2}} u(y)^{1+\frac{4-\sigma}{n-2}} d y
$$

and

$$
J_{1}(r):=\int_{S_{1}(r)}\left(\log \frac{e|y|}{r}\right)|y|^{-n-4+\sigma} f\left(|y|^{n-4} u(y)\right) d y .
$$

Before continuing with the proof of Theorem 1.5, we prove the following lemma.

Lemma 4.1. As $r \rightarrow 0^{+}$we have

$$
\begin{aligned}
& J_{1}(r)=O\left(\log \frac{1}{r}\right), \\
& J_{2}(r)=O\left(\log \frac{1}{r}\right),
\end{aligned}
$$

and

$$
I_{1}(r)=o\left(\log \frac{1}{r}\right)
$$

Proof. By (4.8), (4.7), and (4.5) we have

$$
\begin{aligned}
J_{1}(r) & \leq J_{1}(r)+J_{2}(r) \leq C\left[I_{1}(r)+I_{2}(r)+\log \frac{e}{r}\right] \\
& =C I_{1}(r)+O\left(\log \frac{1}{r}\right) \quad \text { as } \quad r \rightarrow 0^{+} .
\end{aligned}
$$

If $S_{1}(0)=\emptyset$ then $I_{1}(r) \equiv J_{1}(r) \equiv 0$ for $0<r<1$ and thus (4.10) follows from (4.12). Hence we can assume $S_{1}(0) \neq \emptyset$. So for $r$ small and positive, $S_{1}(r) \neq \emptyset, I_{1}(r)>0$, and

$$
\begin{aligned}
J_{1}(r) & \geq\left[\inf _{y \in S_{1}(r)}\left(\log \frac{e|y|}{r}\right)|y|^{-2+\sigma} f\left(|y|^{n-4} u(y)\right)\right] \frac{I_{1}(r)}{M} \\
& \geq\left[\inf _{r<|y|<1}\left(\log \frac{e|y|}{r}\right)\left(|y|^{-2}\right)^{1-\sigma / 2} f\left(M|y|^{-2}\right)\right] \frac{I_{1}(r)}{M}
\end{aligned}
$$


by (3.2) and because $1<|y|^{n-4} u(y) \leq M|y|^{-2}$ for $y \in S_{1}(r)$. Thus

$\frac{M^{2-\sigma / 2} J_{1}(r)}{I_{1}(r)} \geq \min \left\{\inf _{r<|y|<\sqrt{r}}\left(M|y|^{-2}\right)^{1-\sigma / 2} f\left(M|y|^{-2}\right), \quad\left(\log \frac{e}{\sqrt{r}}\right) \inf _{\sqrt{r}<|y|<1}\left(M|y|^{-2}\right)^{1-\sigma / 2} f\left(M|y|^{-2}\right)\right\} \rightarrow \infty$ as $r \rightarrow 0^{+}$by (1.15). Hence (4.12) implies (4.9); and (4.9) implies (4.11). Finally, (4.10) follows from (4.12) and (4.11).

Continuing with the proof of Theorem 1.5, it follows from (4.5), (4.11), and (4.7) that there is a constant $C>0$ such that for $0<r<1$ we have

$$
\begin{aligned}
C & \geq \frac{1}{\log \frac{e}{r}} \int_{r<|y|<1}\left(\log \frac{e|y|}{r}\right)\left(-\Delta^{2} u(y)\right) d y \\
& \geq \frac{1}{\log \frac{e}{r}} \int_{\sqrt{r}<|y|<1}\left(\log \frac{e}{\sqrt{r}}\right)\left(-\Delta^{2} u(y)\right) d y \\
& \geq \frac{1}{2} \int_{\sqrt{r}<|y|<1}-\Delta^{2} u(y) d y .
\end{aligned}
$$

Thus

$$
\int_{|y|<1}-\Delta^{2} u(y) d y<\infty
$$

By (4.11) there exists a constant $C>0$ such that $I_{1}\left(2^{-(j+1)}\right) \leq C(j+1)$ for $j=0,1,2, \ldots$. Thus for each $\varepsilon>0$ we have

$$
\begin{aligned}
M \int_{S_{1}(0)}|x|^{-n-2+\varepsilon} d x & \leq \sum_{j=0}^{\infty} 2^{-\varepsilon j} M \int_{2^{-(j+1)}<|x|<2^{-j}}|x|^{-n-2} d x \\
& \leq \sum_{j=0}^{\infty} 2^{-\varepsilon j} I_{1}\left(2^{-(j+1)}\right) \\
& \leq C \sum_{j=0}^{\infty}(j+1)\left(2^{-\varepsilon}\right)^{j}<\infty
\end{aligned}
$$

Hence, for $0<\varepsilon \leq 1$,

$$
\int_{|x|<1}|x|^{-4+\varepsilon} u(x) d x \leq M \int_{S_{1}(0)}|x|^{-n-2+\varepsilon} d x+\int_{|x|<1}|x|^{-n+\varepsilon} d x<\infty
$$

and so taking $\varepsilon=1$ we have for $0<r<1$ that

$$
\int_{|x|<r} u(x) d x \leq \int_{|x|<r} \frac{r^{3}}{|x|^{3}} u(x) d x=o\left(r^{3}\right) \quad \text { as } \quad r \rightarrow 0^{+} .
$$

Let $F=\Delta^{2} u$ and

$$
\hat{N}(x)=\int_{B_{1}(0)} \Phi(x-y) F(y) d y \quad \text { for } \quad x \in \mathbb{R}^{n} .
$$

By (4.1) and (4.13) we have for $0<r \leq 1$ that

$$
\begin{aligned}
\int_{|x|<r}|\hat{N}(x)| d x & =\int_{|y|<1}\left(\int_{|x|<r} \frac{A}{|x-y|^{n-4}} d x\right)(-F(y)) d y \\
& \leq \int_{|y|<1}\left(\int_{|x|<r} \frac{A}{|x|^{n-4}} d x\right)(-F(y)) d y \\
& =C r^{4}
\end{aligned}
$$


In particular $\hat{N} \in L_{\text {loc }}^{1}\left(B_{1}(0)\right)$. Also for $\varphi \in C_{0}^{\infty}\left(B_{1}(0)\right)$ we have

$$
\begin{aligned}
\int_{B_{1}(0)} \hat{N} \Delta^{2} \varphi d x & =\int_{B_{1}(0)}\left(\int_{B_{1}(0)} \Phi(x-y) \Delta^{2} \varphi(x) d x\right) F(y) d y \\
& =\int_{B_{1}(0)} \varphi(y) F(y) d y .
\end{aligned}
$$

Thus $\Delta^{2} \hat{N}=F$ in $\mathcal{D}^{\prime}\left(B_{1}(0)\right)$.

Let $v=u-\hat{N}$. By (4.14) and (4.15) we see that $v$ satisfies (2.7). Since $\Delta^{2} u=F$ in $\mathcal{D}^{\prime}\left(B_{1}(0) \backslash\right.$ $\{0\})$ we have $\Delta^{2} v=\Delta^{2} u-\Delta^{2} \hat{N}=0$ in $\mathcal{D}^{\prime}\left(B_{1}(0) \backslash\{0\}\right)$. Thus Lemma 2.5 implies for some constant $a$ and some $C^{\infty}$ solution $H$ of $\Delta^{2} H=0$ in $B_{1}(0)$ we have

$$
u=\hat{N}+a \Phi+H \quad \text { in } \quad B_{1}(0) \backslash\{0\} .
$$

Hence, since $\hat{N}<0$ and $u>0$ we have $a \geq 0$.

Case I. Suppose the constant $a$ in (4.16) is positive. By (4.1) we have

$$
\frac{\rho^{-4}}{A\left|\partial B_{1}\right|} \int_{|x|=\rho} \Phi(x-y) d S_{x} \leq \begin{cases}\rho^{-1}, & \text { if }|y|<\rho \\ |y|^{4-n} \rho^{n-5}, & \text { if }|y|>\rho .\end{cases}
$$

Thus for $0<r<1$ we have

$$
\begin{aligned}
\frac{1}{A\left|\partial B_{1}\right|} \int_{r<|x|<1}|x|^{-4}(-\hat{N}(x)) d x & =\int_{|y|<1} \int_{r}^{1} \frac{\rho^{-4}}{A\left|\partial B_{1}\right|} \int_{|x|=\rho} \Phi(x-y) d S_{x} d \rho\left(-\Delta^{2} u(y)\right) d y \\
& \leq \int_{r<|y|<1}\left(\int_{r}^{|y|}|y|^{4-n} \rho^{n-5} d \rho+\int_{|y|}^{1} \rho^{-1} d \rho\right)\left(-\Delta^{2} u(y)\right) d y \\
& +\int_{|y|<r}\left(\int_{r}^{1} \rho^{-1} d \rho\right)\left(-\Delta^{2} u(y)\right) d y \\
& \leq \int_{r<|y|<1}\left(\log \frac{e}{|y|}\right)\left(-\Delta^{2} u(y)\right) d y+\left(\log \frac{1}{r}\right) \int_{|y|<r}-\Delta^{2} u(y) d y \\
& =o\left(\log \frac{e}{r}\right) \text { as } r \rightarrow 0^{+}
\end{aligned}
$$

by (4.13) and the fact that

$$
\begin{aligned}
\int_{r<|y|<1}\left(\log \frac{e}{|y|}\right)\left(-\Delta^{2} u(y)\right) d y & \leq\left(\log \frac{e}{r}\right) \int_{r<|y|<\left(\log \frac{1}{r}\right)^{-1}}-\Delta^{2} u(y) d y \\
& +\left(\log \left(e \log \frac{1}{r}\right)\right) \int_{|y|<1}-\Delta^{2} u(y) d y
\end{aligned}
$$

Hence by (4.16)

$$
\begin{aligned}
\int_{r<|x|<1}|x|^{-4} u(x) d x & =a \int_{r<|x|<1}|x|^{-4} \frac{A}{|x|^{n-4}} d x-o\left(\log \frac{1}{r}\right) \\
& \geq C \log \frac{1}{r} \quad \text { for small } r>0
\end{aligned}
$$


where $C$ is a positive constant. Thus by (4.6) and (4.11) we have

$$
I_{2}(r) \geq C \log \frac{1}{r} \quad \text { for small } r>0 .
$$

On the other hand, by Hölder's inequality and (4.10),

$$
\begin{aligned}
I_{2}(r) & =\int_{S_{2}(r)}|x|^{\frac{2 \sigma-8}{n+2-\sigma}}\left(|x|^{\frac{2 \sigma-4 n}{n+2-\sigma}} u(x)\right) d x \\
& \leq\left(\int_{0<|x|<1}|x|^{-2} d x\right)^{\frac{4-\sigma}{n+2-\sigma}} J_{2}(r)^{\frac{n-2}{n+2-\sigma}} \\
& =O\left(\left(\log \frac{1}{r}\right)^{\frac{n-2}{n+2-\sigma}}\right) \text { as } r \rightarrow 0^{+}
\end{aligned}
$$

which contradicts (4.17) and completes the proof of Theorem 1.5 in Case I.

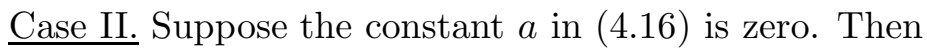

$$
0<u=\hat{N}+H \quad \text { for } \quad 0<|x|<1 .
$$

Thus $-\hat{N}$ and $u$ are positive and bounded for $0<|x| \leq 1 / 2$ and so (3.3) implies

$$
-\Delta^{2} u \geq C|x|^{\frac{2 \sigma-4 n}{n-2}} u^{1+\frac{4-\sigma}{n-2}} \quad \text { in } \overline{B_{1}(0)} \backslash\{0\}
$$

for some positive constant $C$. Also, since (4.1) implies

$$
\begin{aligned}
-\frac{1}{A} \overline{\hat{N}}(r): & =\frac{1}{A\left|\partial B_{r}\right|} \int_{|x|=r}-\hat{N}(x) d S_{x}=\int_{|y|<1} \frac{1}{\left|\partial B_{r}\right|} \int_{|x|=r} \frac{d S_{x}}{|x-y|^{n-4}}(-F(y)) d y \\
& \geq \frac{4}{n} \int_{r<|y|<1}|y|^{4-n}(-F(y)) d y \quad \text { for } \quad 0<r<1 / 2
\end{aligned}
$$

we see that

$$
-\hat{N}_{0}:=\int_{|y|<1} \frac{A}{|y|^{n-4}}(-F(y)) d y<\infty .
$$

Averaging (4.18) we obtain

$$
0<\bar{u}(r)=\overline{\left(\hat{N}-\hat{N}_{0}\right)}(r)+a_{0}-a_{1} A r^{2} \quad \text { for } \quad 0<r<1
$$

for some constants $a_{0}$ and $a_{1}$.

By (4.20), (4.21) and (4.1) we have

$$
\begin{aligned}
& \frac{1}{A} \overline{\left(\hat{N}-\hat{N}_{0}\right)}(r) \\
& =\int_{|y|<1}\left(\frac{1}{\left|\partial B_{r}\right|} \int_{|x|=r} \frac{d S_{x}}{|x-y|^{n-4}}-|y|^{4-n}\right) F(y) d y \\
& =\int_{|y|<r}\left(r^{4-n}-|y|^{4-n}-\frac{n-4}{n} r^{2-n}|y|^{2}\right) F(y) d y-\int_{r<|y|<1} \frac{n-4}{n} r^{2}|y|^{2-n} F(y) d y \\
& =-\int_{|y|<r}|y|^{4-n}\left[1-\left(\frac{|y|}{r}\right)^{n-4}+\frac{n-4}{n}\left(\frac{|y|}{r}\right)^{n-2}\right] F(y) d y \\
& -\int_{r<|y|<\sqrt{r}} \frac{n-4}{n}\left(\frac{r}{|y|}\right)^{2}|y|^{4-n} F(y) d y-\int_{\sqrt{r}<|y|<1} \frac{n-4}{n}\left(\frac{r}{|y|}\right)^{2}|y|^{4-n} F(y) d y \\
& \rightarrow 0 \quad \text { as } \quad r \rightarrow 0^{+}
\end{aligned}
$$


by (4.21). Thus by (4.22) we have $a_{0} \geq 0$. If $a_{0}>0$ then by (3.3) and the boundedness of $u$ in $B_{1}(0)$ we have

$$
\begin{aligned}
-\bar{F}(r) & =-\overline{\Delta^{2} u}(r) \geq r^{\frac{2 \sigma-4 n}{n-2}} \bar{u}(r)^{1+\frac{4-\sigma}{n-2}} \\
& \geq r^{-4-\frac{8-2 \sigma}{n-2}}\left(\frac{a_{0}}{2}\right)^{1+\frac{4-\sigma}{n-2}}
\end{aligned}
$$

for $r$ small and positive and thus for small $r_{0}>0$ we have

$$
\begin{aligned}
\int_{|y|<r_{0}}|y|^{4-n}(-F(y)) d y & =\int_{0}^{r_{0}} r^{4-n} \int_{|y|=r}-F(y) d S_{y} d r \\
& =\left|\partial B_{1}\right| \int_{0}^{r_{0}} r^{3}(-\bar{F}(r)) d r=\infty
\end{aligned}
$$

which contradicts (4.21). So $a_{0}=0, \bar{u}(r) \rightarrow 0$ as $r \rightarrow 0$ and by (4.22) and (4.23) we have

$$
\begin{aligned}
\frac{\bar{u}(r)}{A} & =\frac{\overline{\left(\hat{N}-\hat{N}_{0}\right)}(r)}{A}-a_{1} r^{2} \\
& =\int_{|y|<r}|y|^{4-n}\left[1-\left(\frac{|y|}{r}\right)^{n-4}+\frac{n-4}{n}\left(\frac{|y|}{r}\right)^{n-2}\right]\left(-\Delta^{2} u(y)\right) d y+\left(J(r)-a_{1}\right) r^{2}
\end{aligned}
$$

where

$$
J(r):=\int_{r<|y|<1} \frac{n-4}{n}|y|^{2-n}\left(-\Delta^{2} u(y)\right) d y \quad \text { for } \quad 0 \leq r<1 .
$$

$(J(0)$ may be $\infty$.)

Case II(a). Suppose $a_{1}<J(0)$. Then there exists $\varepsilon>0$ and $r_{0} \in(0,1)$ such that $a_{1} \leq(1-\varepsilon) J(r)$ for $0<r \leq r_{0}$. Thus by (4.24), $\frac{\bar{u}(r)}{A} \geq \varepsilon r^{2} J(r)$ for $0<r<r_{0}$, which together with (4.19) and Lemma 2.6 gives a contradiction and thereby proves Theorem 1.5 when $n \geq 5$ in Case II(a).

Case II(b). Suppose $a_{1} \geq J(0)$. Then for $0<r<1$ we have

$$
\left(J(r)-a_{1}\right) r^{2} \leq(J(r)-J(0)) r^{2}=-\int_{|y|<r} \frac{n-4}{n} r^{2}|y|^{2-n}\left(-\Delta^{2} u(y)\right) d y
$$

and hence by (4.24) we have

$$
\begin{aligned}
0<\frac{\bar{u}(r)}{A} & \leq \int_{|y|<r}|y|^{4-n}\left[1-\left(\frac{r}{|y|}\right)^{4-n}+\frac{n-4}{n}\left(\left(\frac{r}{|y|}\right)^{2-n}-\left(\frac{r}{|y|}\right)^{2}\right)\right]\left(-\Delta^{2} u(y)\right) d y \\
& =-\int_{|y|<r}|y|^{2} r^{2-n} p\left(\frac{r}{|y|}\right)\left(-\Delta^{2} u(y)\right) d y
\end{aligned}
$$

where $p(t):=\frac{n-4}{n} t^{n}-t^{n-2}+t^{2}-\frac{n-4}{n}$. Since $p(1)=p^{\prime}(1)=p^{\prime \prime}(1)=0$ and

$$
p^{\prime \prime \prime}(t)=(n-4)(n-2)(n-1) t^{n-5}\left(t^{2}-\frac{n-3}{n-1}\right)>0 \quad \text { for } \quad t \geq 1
$$

we see that $p(t)>0$ for $t>1$. This contradicts (4.25) and completes the proof of Theorem 1.5 when $n \geq 5$ in all cases. 


\section{Completion of the Proof of Theorem 1.5 when $n=4$}

When $n=4$, we complete in this section the proof of Theorem 1.5 which we began in Section 3 . Completion of the proof of Theorem 1.5 when $n=4$. For $x \in \mathbb{R}^{4}$ we see by Lemma 2.1 that

$$
\frac{1}{\left|\partial B_{r}\right|} \int_{|y|=r} \log \frac{e}{|x-y|} d S_{y}= \begin{cases}\log \frac{e}{r}-\frac{1}{4} r^{-2}|x|^{2}, & \text { if }|x|<r \\ \log \frac{e}{|x|}-\frac{1}{4} r^{2}|x|^{-2}, & \text { if }|x|>r .\end{cases}
$$

It therefore follows from equations (1.11) and (A.1) that for $r>0$ we have

$$
\frac{1}{A\left|\partial B_{r}\right|} \int_{|x|=r} \Psi(x, y) d S_{x}= \begin{cases}-\frac{1}{4} r^{-2}|y|^{2}, & \text { if }|y|<r \\ \left(\log \frac{r}{e|y|}\right) p\left(\frac{r}{|y|}\right), & \text { if }|y| \geq r\end{cases}
$$

where $p(t):=\left((\log t)-t^{2} / 4\right) / \log (t / e)$ is bounded between positive constants for $0<t \leq 1$. Hence

$$
-\int_{|x|=r} \Psi(x, y) d S_{x} \sim\left\{\begin{array}{ll}
2 r|y|^{2}, & \text { if }|y|<r \\
2 r^{3} \log \frac{e|y|}{r}, & \text { if }|y| \geq r
\end{array} \quad \text { for } \quad(r, y) \in(0, \infty) \times \mathbb{R}^{n} .\right.
$$

Thus by (A.6), for $0<r \leq \frac{1}{4}$, we have

$$
\begin{aligned}
\int_{r<|x|<1}|x|^{-4} N(x) d x & =\int_{|y|<1} \int_{r}^{1} \rho^{-4} \int_{|x|=\rho}-\Psi(x, y) d S_{x} d \rho\left(-\Delta^{2} u(y)\right) d y \\
& \sim \int_{r<|y|<1}\left(\int_{|y|}^{1} 2 \rho^{-3}|y|^{2} d \rho+\int_{r}^{|y|} 2 \rho^{-1} \log \frac{e|y|}{\rho} d \rho\right)\left(-\Delta^{2} u(y)\right) d y \\
& +\int_{|y|<r}\left(\int_{r}^{1} 2 \rho^{-3}|y|^{2} d \rho\right)\left(-\Delta^{2} u(y)\right) d y \\
& \sim \int_{r<|y|<1}\left(\log \frac{e|y|}{r}\right)^{2}\left(-\Delta^{2} u(y)\right) d y+g(r)
\end{aligned}
$$

by Lemma 2.2 with $\alpha=2$ where

$$
0<g(r):=\left(\frac{1}{r^{2}}-1\right) \int_{|y|<r}|y|^{2}\left(-\Delta^{2} u(y)\right) d y=o\left(\frac{1}{r^{2}}\right) \quad \text { as } \quad r \rightarrow 0^{+}
$$

by (A.4).

Let $\varphi(t, r)=t^{-2}\left(\log \frac{e t}{r}\right)^{2}$. Since $\varphi_{t}(t, r) \leq 0$ for $t \geq r>0$, we see for $0<r<1$ that

$$
\begin{aligned}
\int_{r<|y|<1}\left(\log \frac{e|y|}{r}\right)^{2} & \left(-\Delta^{2} u(y)\right) d y=\int_{r<|y|<1} \varphi(|y|, r)|y|^{2}\left(-\Delta^{2} u(y)\right) d y \\
& \leq \varphi(r, r) \int_{r<|y|<\sqrt{r}}|y|^{2}\left(-\Delta^{2} u(y)\right) d y+\varphi(\sqrt{r}, r) \int_{\sqrt{r}<|y|<1}|y|^{2}\left(-\Delta^{2} u(y)\right) d y \\
& \leq \frac{1}{r^{2}} \int_{r<|y|<\sqrt{r}}|y|^{2}\left(-\Delta^{2} u(y)\right) d y+\frac{1}{r}\left(\log \frac{e}{\sqrt{r}}\right)^{2} \int_{|y|<1}|y|^{2}\left(-\Delta^{2} u(y)\right) d y \\
& =o\left(r^{-2}\right) \quad \text { as } r \rightarrow 0^{+}
\end{aligned}
$$


by (A.4). It follows therefore from (5.2) and (5.3) that

$$
\int_{r<|x|<1}|x|^{-4} N(x) d x=o\left(r^{-2}\right) \quad \text { as } \quad r \rightarrow 0^{+} .
$$

Hence, by (3.4) and the positivity of $u$ we see that the constant $c_{4}$ in (3.4) is nonnegative and thus by (3.4), (5.2), and the positivity of $g$ we have

$$
\int_{r<|x|<1}|x|^{-4} u(x) d x \geq \frac{1}{C} \int_{r<|y|<1}\left(\log \frac{e|y|}{r}\right)^{2}\left(-\Delta^{2} u(y)\right) d y-C\left(\log \frac{e}{r}\right)^{2} \quad \text { for } \quad 0<r<\frac{1}{4}
$$

where $C$ is a positive constant independent of $r$.

By (A.3) there exists a constant $M>1$ such that $0<u(x) \leq M|x|^{-2}$ for $0<|x| \leq 1$. Define $I_{1}, I_{2}:(0,1) \rightarrow[0, \infty)$ by

$$
I_{1}(r):=M \int_{x \in S_{1}(r)}|x|^{-6} d x \quad \text { and } \quad I_{2}(r):=\int_{x \in S_{2}(r)}|x|^{-4} u(x) d x
$$

where

$$
S_{1}(r):=\left\{x \in \mathbb{R}^{4}: r<|x|<1 \text { and } 1<u(x) \leq M|x|^{-2}\right\}
$$

and

$$
S_{2}(r):=\left\{x \in \mathbb{R}^{4}: r<|x|<1 \text { and } 0<u(x) \leq 1\right\} .
$$

Then $S_{1}(r) \cup S_{2}(r)=B_{1}(0)-\overline{B_{r}(0)}$,

$$
I_{2}(r)=O\left(\log \frac{1}{r}\right) \quad \text { as } \quad r \rightarrow 0^{+},
$$

and for $0<r<\frac{1}{4}$ we have

$$
\begin{aligned}
I_{1}(r)+I_{2}(r) & \geq \int_{r<|x|<1}|x|^{-4} u(x) d x \\
& \geq \frac{1}{C} \int_{r<|y|<1}\left(\log \frac{e|y|}{r}\right)^{2}\left(-\Delta^{2} u(y)\right) d y-C\left(\log \frac{e}{r}\right)^{2}
\end{aligned}
$$

by (5.4) .

By (3.3) we have

$$
\int_{r<|y|<1}\left(\log \frac{e|y|}{r}\right)^{2}\left(-\Delta^{2} u(y)\right) d y \geq J_{1}(r)+J_{2}(r) \quad \text { for } \quad 0<r<1
$$

where

$$
J_{2}(r):=\int_{S_{2}(r)}|y|^{\sigma-8} u(y)^{3-\sigma / 2} d y
$$

and

$$
J_{1}(r):=\int_{S_{1}(r)}\left(\log \frac{e|y|}{r}\right)^{2}|y|^{\sigma-8} f(u(y)) d y .
$$

Before continuing with the proof of Theorem 1.5, we prove the following lemma. 
Lemma 5.1. As $r \rightarrow 0^{+}$we have

$$
\begin{aligned}
& J_{1}(r)=O\left(\left(\log \frac{1}{r}\right)^{2}\right), \\
& J_{2}(r)=O\left(\left(\log \frac{1}{r}\right)^{2}\right),
\end{aligned}
$$

and

$$
I_{1}(r)=o\left(\left(\log \frac{1}{r}\right)^{2}\right)
$$

Proof. By (5.8), (5.7), and (5.5) we have

$$
\begin{aligned}
J_{1}(r) & \leq J_{1}(r)+J_{2}(r) \leq C\left[I_{1}(r)+I_{2}(r)+\left(\log \frac{e}{r}\right)^{2}\right] \\
& =C I_{1}(r)+O\left(\left(\log \frac{1}{r}\right)^{2}\right) \quad \text { as } \quad r \rightarrow 0^{+} .
\end{aligned}
$$

If $S_{1}(0)=\emptyset$ then $I_{1}(r) \equiv J_{1}(r) \equiv 0$ for $0<r<1$ and thus (5.10) follows from (5.12). Hence we can assume $S_{1}(0) \neq \emptyset$. So for $r$ small and positive, $S_{1}(r) \neq \emptyset, I_{1}(r)>0$, and

$$
\begin{aligned}
J_{1}(r) & \geq\left[\inf _{y \in S_{1}(r)}\left(\log \frac{e|y|}{r}\right)^{2}|y|^{\sigma-2} f(u(y))\right] \frac{I_{1}(r)}{M} \\
& \geq\left[\inf _{r<|y|<1}\left(\log \frac{e|y|}{r}\right)^{2}\left(|y|^{-2}\right)^{1-\sigma / 2} f\left(M|y|^{-2}\right)\right] \frac{I_{1}(r)}{M}
\end{aligned}
$$

by (3.2) and because $1<u(y) \leq M|y|^{-2}$ for $y \in S_{1}(r)$. Thus

$$
\frac{M^{2-\sigma / 2} J_{1}(r)}{I_{1}(r)} \geq \min \left\{\inf _{r<|y|<\sqrt{r}}\left(M|y|^{-2}\right)^{1-\sigma / 2} f\left(M|y|^{-2}\right), \quad\left(\log \frac{e}{\sqrt{r}}\right)^{2} \inf _{\sqrt{r}<|y|<1}\left(M|y|^{-2}\right)^{1-\sigma / 2} f\left(M|y|^{-2}\right)\right\} \rightarrow \infty
$$

as $r \rightarrow 0^{+}$by (1.15). Hence (5.12) implies (5.9); and (5.9) implies (5.11). Finally, (5.10) follows from (5.12) and (5.11).

Continuing with the proof of Theorem 1.5, it follows from (5.5), (5.11), and (5.7) that there is a constant $C>0$ such that for $0<r<1$ we have

$$
\begin{aligned}
C & \geq \frac{1}{\left(\log \frac{e}{r}\right)^{2}} \int_{r<|y|<1}\left(\log \frac{e|y|}{r}\right)^{2}\left(-\Delta^{2} u(y)\right) d y \\
& \geq \frac{1}{\left(\log \frac{e}{r}\right)^{2}} \int_{\sqrt{r}<|y|<1}\left(\log \frac{e}{\sqrt{r}}\right)^{2}\left(-\Delta^{2} u(y)\right) d y \\
& \geq \frac{1}{4} \int_{\sqrt{r}<|y|<1}-\Delta^{2} u(y) d y
\end{aligned}
$$

Thus

$$
\int_{|y|<1}-\Delta^{2} u(y) d y<\infty
$$


By (5.11) there exists a constant $C>0$ such that $I_{1}\left(2^{-(j+1)}\right) \leq C(j+1)^{2}$ for $j=0,1,2, \ldots$ Thus for each $\varepsilon>0$ we have

$$
\begin{aligned}
M \int_{S_{1}(0)}|x|^{-6+\varepsilon} d x & \leq \sum_{j=0}^{\infty} 2^{-\varepsilon j} M \int_{\substack{2^{-(j+1)}<|x|<S_{1}(0) \\
x \in j}}|x|^{-6} d x \\
& \leq \sum_{j=0}^{\infty} 2^{-\varepsilon j} I_{1}\left(2^{-(j+1)}\right) \\
& \leq C \sum_{j=0}^{\infty}(j+1)^{2}\left(2^{-\varepsilon}\right)^{j}<\infty
\end{aligned}
$$

Hence, for $0<\varepsilon \leq 1$,

$$
\int_{|x|<1}|x|^{-4+\varepsilon} u(x) d x \leq M \int_{S_{1}(0)}|x|^{-6+\varepsilon} d x+\int_{|x|<1}|x|^{-4+\varepsilon} d x<\infty
$$

and so taking $\varepsilon=1$ we have for $0<r<1$ that

$$
\int_{|x|<r} u(x) d x \leq \int_{|x|<r} \frac{r^{3}}{|x|^{3}} u(x) d x=o\left(r^{3}\right) \quad \text { as } \quad r \rightarrow 0^{+} .
$$

Let $F=\Delta^{2} u$ and

$$
\hat{N}(x)=\int_{B_{1}(0)} \Phi(x-y) F(y) d y \quad \text { for } \quad x \in \mathbb{R}^{4} .
$$

By (5.1) and (5.13) we have for $0<r \leq 1$ that

$$
\begin{aligned}
\int_{|x|<r}|\hat{N}(x)| d x & =\int_{|y|<1}\left(\int_{|x|<r} A \log \frac{e}{|x-y|} d x\right)(-F(y)) d y \\
& \leq \int_{|y|<1}\left(\int_{|x|<r} A \log \frac{e}{|x|} d x\right)(-F(y)) d y \\
& \leq C r^{4} \log \frac{e}{r} .
\end{aligned}
$$

In particular $\hat{N} \in L_{\text {loc }}^{1}\left(B_{1}(0)\right)$. Also for $\varphi \in C_{0}^{\infty}\left(B_{1}(0)\right)$ we have

$$
\begin{aligned}
\int_{B_{1}(0)} \hat{N} \Delta^{2} \varphi d x & =\int_{B_{1}(0)}\left(\int_{B_{1}(0)} \Phi(x-y) \Delta^{2} \varphi(x) d x\right) F(y) d y \\
& =\int_{B_{1}(0)} \varphi(y) F(y) d y .
\end{aligned}
$$

Thus $\Delta^{2} \hat{N}=F$ in $\mathcal{D}^{\prime}\left(B_{1}(0)\right)$.

Let $v=u-\hat{N}$. By (5.14) and (5.15) we see that $v$ satifies (2.7). Since $\Delta^{2} u=F$ in $\mathcal{D}^{\prime}\left(B_{1}(0) \backslash\{0\}\right)$ we have $\Delta^{2} v=\Delta^{2} u-\Delta^{2} \hat{N}=0$ in $\mathcal{D}^{\prime}\left(B_{1}(0) \backslash\{0\}\right)$. Thus Lemma 2.5 implies for some constant $a$ and some $C^{\infty}$ solution $H$ of $\Delta^{2} H=0$ in $B_{1}(0)$ we have

$$
u=\hat{N}+a \Phi+H \quad \text { in } \quad B_{1}(0) \backslash\{0\} .
$$

Hence, since $\hat{N}<0$ and $u>0$ we have $a \geq 0$. 
Case I. Suppose the constant $a$ in (5.16) is positive. By (5.1) we have

$$
\frac{\rho^{-4}}{A\left|\partial B_{1}\right|} \int_{|x|=\rho} \Phi(x-y) d S_{x} \leq \begin{cases}\rho^{-1} \log \frac{e}{\rho}, & \text { if }|y|<\rho \\ \rho^{-1} \log \frac{e}{|y|}, & \text { if }|y|>\rho .\end{cases}
$$

Thus for $0<r<1$ we have

$$
\begin{aligned}
\frac{1}{A\left|\partial B_{1}\right|} \int_{r<|x|<1}|x|^{-4} & (-\hat{N}(x)) d x=\int_{|y|<1} \int_{r}^{1} \frac{\rho^{-4}}{A\left|\partial B_{1}\right|} \int_{|x|=\rho} \Phi(x-y) d S_{x} d \rho\left(-\Delta^{2} u(y)\right) d y \\
& \leq \int_{r<|y|<1}\left(\int_{r}^{|y|} \rho^{-1} \log \frac{e}{|y|} d \rho+2 \int_{|y|}^{1} \rho^{-1} \log \frac{e}{\rho} d \rho\right)\left(-\Delta^{2} u(y)\right) d y \\
& +\int_{|y|<r}\left(\int_{r}^{1} \rho^{-1} \log \frac{e}{\rho} d \rho\right)\left(-\Delta^{2} u(y)\right) d y \\
& \leq\left(\log \frac{e}{r}\right) \int_{r<|y|<1}\left(\log \frac{e}{|y|}\right)\left(-\Delta^{2} u(y)\right) d y+\left(\log \frac{e}{r}\right)^{2} \int_{|y|<r}-\Delta^{2} u(y) d y \\
& =o\left(\left(\log \frac{e}{r}\right)^{2}\right) \text { as } r \rightarrow 0^{+}
\end{aligned}
$$

by $(5.13)$ and the fact that

$$
\begin{aligned}
\int_{r<|y|<1}\left(\log \frac{e}{|y|}\right)\left(-\Delta^{2} u(y)\right) d y & \leq\left(\log \frac{e}{r}\right) \int_{r<|y|<\left(\log \frac{1}{r}\right)^{-1}}-\Delta^{2} u(y) d y \\
& +\left(\log \left(e \log \frac{1}{r}\right)\right) \int_{|y|<1}-\Delta^{2} u(y) d y
\end{aligned}
$$

Hence by (5.16)

$$
\begin{aligned}
\int_{r<|x|<1}|x|^{-4} u(x) d x & =a \int_{r<|x|<1}|x|^{-4} A \log \frac{e}{|x|} d x-o\left(\left(\log \frac{1}{r}\right)^{2}\right) \\
& \geq C\left(\log \frac{1}{r}\right)^{2} \quad \text { for small } r>0
\end{aligned}
$$

where $C$ is a positive constant. Thus by (5.6) and (5.11) we have

$$
I_{2}(r) \geq C\left(\log \frac{1}{r}\right)^{2} \quad \text { for small } r>0 .
$$

On the other hand, by Hölder's inequality and (15.10),

$$
\begin{aligned}
I_{2}(r) & =\int_{S_{2}(r)}|x|^{\frac{2 \sigma-8}{6-\sigma}}\left(|x|^{\frac{2 \sigma-16}{6-\sigma}} u(x)\right) d x \\
& \leq\left(\int_{0<|x|<1}|x|^{-2} d x\right)^{\frac{4-\sigma}{6-\sigma}} J_{2}(r)^{\frac{2}{6-\sigma}} \\
& =O\left(\left(\log \frac{1}{r}\right)^{\frac{4}{6-\sigma}}\right) \quad \text { as } r \rightarrow 0^{+}
\end{aligned}
$$


which contradicts (5.17) and completes the proof of Theorem 1.5 in Case I.

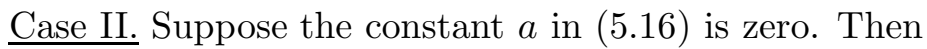

$$
0<u=\hat{N}+H \quad \text { for } \quad 0<|x|<1 .
$$

Thus $-\hat{N}$ and $u$ are positive and bounded for $0<|x| \leq 1 / 2$ and so (3.3) implies

$$
-\Delta^{2} u \geq C|x|^{\sigma-8} u^{3-\sigma / 2} \quad \text { in } \overline{B_{1}(0)} \backslash\{0\}
$$

for some positive constant $C$. Also, since (5.1) implies

$$
\begin{aligned}
-\frac{1}{A} \overline{\hat{N}}(r): & =\frac{1}{A\left|\partial B_{r}\right|} \int_{|x|=r}-\hat{N}(x) d S_{x}=\int_{|y|<1} \frac{1}{\left|\partial B_{r}\right|} \int_{|x|=r} \log \frac{e}{|x-y|} d S_{x}(-F(y)) d y \\
& \geq \frac{3}{4} \int_{r<|y|<1}\left(\log \frac{e}{|y|}\right)(-F(y)) d y \quad \text { for } \quad 0<r<1 / 2
\end{aligned}
$$

we see that

$$
-\hat{N}_{0}:=\int_{|y|<1} A\left(\log \frac{e}{|y|}\right)(-F(y)) d y<\infty .
$$

Averaging (5.18) we obtain

$$
0<\bar{u}(r)=\overline{\left(\hat{N}-\hat{N}_{0}\right)}(r)+a_{0}-a_{1} A r^{2} \quad \text { for } \quad 0<r<1
$$

for some constants $a_{0}$ and $a_{1}$.

By (5.20), (5.21) and (5.1) we have

$$
\begin{aligned}
& \frac{1}{A} \overline{\left(\hat{N}-\hat{N}_{0}\right)}(r) \\
& =\int_{|y|<1}\left(\frac{1}{\left|\partial B_{r}\right|} \int_{|x|=r} \log \frac{e}{|x-y|} d S_{x}-\log \frac{e}{|y|}\right) F(y) d y \\
& =\int_{|y|<r}\left(\log \frac{e}{r}-\log \frac{e}{|y|}-\frac{1}{4}\left(\frac{|y|}{r}\right)^{2}\right) F(y) d y-\int_{r<|y|<1} \frac{1}{4} r^{2}|y|^{-2} F(y) d y \\
& =-\int_{|y|<r}\left(\log \frac{e}{|y|}\right)\left[1-\frac{\log \frac{e}{r}}{\log \frac{e}{|y|}}+\frac{1}{4} \frac{\left(\frac{|y|}{r}\right)^{2}}{\log \frac{e}{|y|}}\right] F(y) d y \\
& -\int_{r<|y|<\sqrt{r}} \frac{1}{4} \frac{\left(\frac{r}{|y|}\right)^{2}}{\log \frac{e}{|y|}}\left(\log \frac{e}{|y|}\right) F(y) d y-\int_{\sqrt{r}<|y|<1} \frac{1}{4} \frac{\left(\frac{r}{|y|}\right)^{2}}{\log \frac{e}{|y|}}\left(\log \frac{e}{|y|}\right) F(y) d y \\
& \rightarrow 0 \quad \text { as } \quad r \rightarrow 0^{+}
\end{aligned}
$$

by (5.21). Thus by (5.22) we have $a_{0} \geq 0$. If $a_{0}>0$ then by (5.19) we have

$$
\begin{aligned}
-\bar{F}(r) & =-\overline{\Delta^{2} u}(r) \geq C r^{\sigma-8} \bar{u}(r)^{3-\sigma / 2} \\
& \geq C r^{\sigma-8}\left(\frac{a_{0}}{2}\right)^{3-\sigma / 2}
\end{aligned}
$$


for $r$ small and positive and thus for small $r_{0}>0$ we have

$$
\begin{aligned}
\int_{|y|<r_{0}}\left(\log \frac{e}{|y|}\right)(-F(y)) d y & =\int_{0}^{r_{0}}\left(\log \frac{e}{r}\right) \int_{|y|=r}-F(y) d S_{y} d r \\
& =\left|\partial B_{1}\right| \int_{0}^{r_{0}} r^{3}\left(\log \frac{e}{r}\right)(-\bar{F}(r)) d r=\infty
\end{aligned}
$$

which contradicts (5.21). So $a_{0}=0, \bar{u}(r) \rightarrow 0$ as $r \rightarrow 0$ and by (5.22) and (5.23) we have

$$
\begin{aligned}
\frac{\bar{u}(r)}{A} & =\frac{\overline{\left(\hat{N}-\hat{N}_{0}\right)}(r)}{A}-a_{1} r^{2} \\
& =\int_{|y|<r}\left(\log \frac{e}{|y|}\right)\left[1-\frac{\log \frac{e}{r}}{\log \frac{e}{|y|}}+\frac{1}{4} \frac{\left(\frac{|y|}{r}\right)^{2}}{\log \frac{e}{|y|}}\right]\left(-\Delta^{2} u(y)\right) d y+\left(J(r)-a_{1}\right) r^{2}
\end{aligned}
$$

where

$$
J(r):=\int_{r<|y|<1} \frac{1}{4}|y|^{-2}\left(-\Delta^{2} u(y)\right) d y \quad \text { for } \quad 0 \leq r<1 .
$$

$(J(0)$ may be $\infty$.)

Case II(a). Suppose $a_{1}<J(0)$. Then there exists $\varepsilon>0$ and $r_{0} \in(0,1)$ such that $a_{1} \leq(1-\varepsilon) J(r)$

for $0<r \leq r_{0}$. Thus by (5.24), $\frac{\bar{u}(r)}{A} \geq \varepsilon r^{2} J(r)$ for $0<r<r_{0}$, which together with (5.19) and Lemma 2.6 gives a contradiction and thereby proves Theorem 1.5 when $n=4$ in Case II(a).

Case II(b). Suppose $a_{1} \geq J(0)$. Then for $0<r<1$ we have

$$
\left(J(r)-a_{1}\right) r^{2} \leq(J(r)-J(0)) r^{2}=-\int_{|y|<r} \frac{1}{4} r^{2}|y|^{-2}\left(-\Delta^{2} u(y)\right) d y
$$

and hence by (5.24) we have

$$
\begin{aligned}
0<\frac{\bar{u}(r)}{A} & \leq \int_{|y|<r}\left(\log \frac{r}{|y|}+\frac{1}{4}\left(\frac{|y|}{r}\right)^{2}-\frac{1}{4}\left(\frac{r}{|y|}\right)^{2}\right)\left(-\Delta^{2} u(y)\right) d y \\
& =-\int_{|y|<r}|y|^{2} r^{-2} p\left(\frac{r}{|y|}\right)\left(-\Delta^{2} u(y)\right) d y
\end{aligned}
$$

where $p(t):=\frac{1}{4} t^{4}-t^{2} \log t-\frac{1}{4}$. Since $p(1)=p^{\prime}(1)=p^{\prime \prime}(1)=0$ and

$$
p^{\prime \prime \prime}(t)=6 t^{-1}\left(t^{2}-\frac{1}{3}\right)>0 \quad \text { for } \quad t \geq 1
$$

we see that $p(t)>0$ for $t>1$. This contradicts (5.25) and completes the proof of Theorem 1.5 when $n=4$ in all cases.

\section{Completion of the Proof of Theorem 1.5 when $n=3$}

When $n=3$, we complete in this section the proof of Theorem 1.5 which we began in Section 3 . 
Completion of the proof of Theorem 1.5 when $n=3$. For $x \in \mathbb{R}^{3}$ we see by Lemma 2.1 that

$$
\frac{1}{\left|\partial B_{r}\right|} \int_{|y|=r}|x-y| d S_{y}= \begin{cases}r+\frac{1}{3} r^{-1}|x|^{2}, & \text { if }|x|<r \\ |x|+\frac{1}{3} r^{2}|x|^{-1}, & \text { if }|x|>r\end{cases}
$$

It therefore follows from equations (1.12) and (A.1) that for $r>0$ we have

$$
\frac{-1}{A\left|\partial B_{r}\right|} \int_{|x|=r} \Psi(x, y) d S_{x}= \begin{cases}\frac{1}{3} r^{-1}|y|^{2}, & \text { if }|y|<r \\ |y| p\left(\frac{r}{|y|}\right), & \text { if }|y| \geq r\end{cases}
$$

where $p(t):=1-t+\frac{1}{3} t^{2}$ is bounded between positive constants for $0 \leq t \leq 1$. Hence

$$
-\int_{|x|=r} \Psi(x, y) d S_{x} \sim\left\{\begin{array}{ll}
2 r|y|^{2}, & \text { if }|y|<r \\
r^{2}|y|, & \text { if }|y| \geq r
\end{array} \quad \text { for } \quad(r, y) \in(0, \infty) \times \mathbb{R}^{3} .\right.
$$

Thus by (A.6), for $0<r \leq \frac{1}{4}$, we have

$$
\begin{aligned}
\int_{r<|x|<1}|x|^{-4} N(x) d x & =\int_{|y|<1} \int_{r}^{1} \rho^{-4} \int_{|x|=\rho}-\Psi(x, y) d S_{x} d \rho\left(-\Delta^{2} u(y)\right) d y \\
& \sim \int_{r<|y|<1}\left(\int_{|y|}^{1} 2 \rho^{-3}|y|^{2} d \rho+\int_{r}^{|y|} \rho^{-2}|y| d \rho\right)\left(-\Delta^{2} u(y)\right) d y \\
& +\int_{|y|<r}\left(\int_{r}^{1} 2 \rho^{-3}|y|^{2} d \rho\right)\left(-\Delta^{2} u(y)\right) d y \\
& \sim \int_{r<|y|<1}\left(\frac{|y|}{r}\right)\left(-\Delta^{2} u(y)\right) d y+g(r)
\end{aligned}
$$

where

$$
0<g(r):=\left(\frac{1}{r^{2}}-1\right) \int_{|y|<r}|y|^{2}\left(-\Delta^{2} u(y)\right) d y=o\left(\frac{1}{r^{2}}\right) \quad \text { as } \quad r \rightarrow 0^{+}
$$

by (A.4).

For $0<r<1$ we have

$$
\begin{aligned}
\int_{r<|y|<1}\left(\frac{|y|}{r}\right) & \left(-\Delta^{2} u(y)\right) d y=\int_{r<|y|<1} \frac{1}{r|y|}|y|^{2}\left(-\Delta^{2} u(y)\right) d y \\
& \leq \frac{1}{r^{2}} \int_{r<|y|<\sqrt{r}}|y|^{2}\left(-\Delta^{2} u(y)\right) d y+\frac{1}{r^{3 / 2}} \int_{\sqrt{r}<|y|<1}|y|^{2}\left(-\Delta^{2} u(y)\right) d y \\
& \leq \frac{1}{r^{2}} \int_{r<|y|<\sqrt{r}}|y|^{2}\left(-\Delta^{2} u(y)\right) d y+\frac{1}{r^{3 / 2}} \int_{|y|<1}|y|^{2}\left(-\Delta^{2} u(y)\right) d y \\
& =o\left(r^{-2}\right) \text { as } r \rightarrow 0^{+}
\end{aligned}
$$

by (A.4). It follows therefore from (6.3) and (6.4) that

$$
\int_{r<|x|<1}|x|^{-4} N(x) d x=o\left(r^{-2}\right) \quad \text { as } \quad r \rightarrow 0^{+} .
$$

Hence, by (3.4) and the positivity of $u$ we see that the constant $c_{4}$ in (3.4) is nonnegative and thus by (3.4), (6.3), and the positivity of $g$ we have

$$
\int_{r<|x|<1}|x|^{-4} u(x) d x \geq \frac{1}{C} \int_{r<|y|<1}\left(\frac{|y|}{r}\right)\left(-\Delta^{2} u(y)\right) d y-C \frac{1}{r} \quad \text { for } \quad 0<r<\frac{1}{4}
$$


where $C$ is a positive constant independent of $r$.

By (A.3) there exists a constant $M>1$ such that $0<u(x) \leq M|x|^{-1}$ for $0<|x| \leq 1$. Define $I_{1}, I_{2}:(0,1) \rightarrow[0, \infty)$ by

$$
I_{1}(r):=M \int_{x \in S_{1}(r)}|x|^{-5} d x \quad \text { and } \quad I_{2}(r):=\int_{x \in S_{2}(r)}|x|^{-4} u(x) d x
$$

where

$$
S_{1}(r):=\left\{x \in \mathbb{R}^{3}: r<|x|<1 \text { and }|x|<u(x) \leq M|x|^{-1}\right\}
$$

and

$$
S_{2}(r):=\left\{x \in \mathbb{R}^{3}: r<|x|<1 \text { and } 0<u(x) \leq|x|\right\} .
$$

Then $S_{1}(r) \cup S_{2}(r)=B_{1}(0)-\overline{B_{r}(0)}$,

$$
I_{2}(r)=O\left(\log \frac{1}{r}\right) \quad \text { as } \quad r \rightarrow 0^{+},
$$

and for $0<r<\frac{1}{4}$ we have

$$
\begin{aligned}
I_{1}(r)+I_{2}(r) & \geq \int_{r<|x|<1}|x|^{-4} u(x) d x \\
& \geq \frac{1}{C} \int_{r<|y|<1}\left(\frac{|y|}{r}\right)\left(-\Delta^{2} u(y)\right) d y-C \frac{1}{r}
\end{aligned}
$$

by $(\underline{6.5})$.

By (3.3) we have

$$
\int_{r<|y|<1}\left(\frac{|y|}{r}\right)\left(-\Delta^{2} u(y)\right) d y \geq J_{1}(r)+J_{2}(r) \quad \text { for } \quad 0<r<1
$$

where

$$
J_{2}(r):=\int_{S_{2}(r)}|y|^{2 \sigma-12} u(y)^{5-\sigma} d y
$$

and

$$
J_{1}(r):=\int_{S_{1}(r)}\left(\frac{|y|}{r}\right)|y|^{\sigma-7} f\left(|y|^{-1} u(y)\right) d y .
$$

Before continuing with the proof of Theorem 1.5, we prove the following lemma.

Lemma 6.1. As $r \rightarrow 0^{+}$we have

$$
\begin{aligned}
& J_{1}(r)=O\left(\frac{1}{r}\right), \\
& J_{2}(r)=O\left(\frac{1}{r}\right),
\end{aligned}
$$

and

$$
I_{1}(r)=o\left(\frac{1}{r}\right) .
$$


Proof. By (6.9), (6.8), and (6.6) we have

$$
\begin{aligned}
J_{1}(r) & \leq J_{1}(r)+J_{2}(r) \leq C\left[I_{1}(r)+I_{2}(r)+\frac{1}{r}\right] \\
& =C I_{1}(r)+O\left(\frac{1}{r}\right) \quad \text { as } \quad r \rightarrow 0^{+} .
\end{aligned}
$$

If $S_{1}(0)=\emptyset$ then $I_{1}(r) \equiv J_{1}(r) \equiv 0$ for $0<r<1$ and thus (6.11) follows from (6.13). Hence we can assume $S_{1}(0) \neq \emptyset$. So for $r$ small and positive, $S_{1}(r) \neq \emptyset, I_{1}(r)>0$, and

$$
\begin{aligned}
J_{1}(r) & \geq\left[\inf _{y \in S_{1}(r)}\left(\frac{|y|}{r}\right)|y|^{\sigma-2} f\left(|y|^{-1} u(y)\right)\right] \frac{I_{1}(r)}{M} \\
& \geq\left[\inf _{r<|y|<1}\left(\frac{|y|}{r}\right)\left(|y|^{-2}\right)^{1-\sigma / 2} f\left(M|y|^{-2}\right)\right] \frac{I_{1}(r)}{M}
\end{aligned}
$$

by (3.2) and because $1<|y|^{-1} u(y) \leq M|y|^{-2}$ for $y \in S_{1}(r)$. Thus

$\frac{M^{2-\sigma / 2} J_{1}(r)}{I_{1}(r)} \geq \min \left\{\inf _{r<|y|<\sqrt{r}}\left(M|y|^{-2}\right)^{1-\sigma / 2} f\left(M|y|^{-2}\right),\left(\frac{1}{\sqrt{r}}\right) \inf _{\sqrt{r}<|y|<1}\left(M|y|^{-2}\right)^{1-\sigma / 2} f\left(M|y|^{-2}\right)\right\} \rightarrow \infty$

as $r \rightarrow 0^{+}$by (1.15). Hence (6.13) implies (6.10); and (6.10) implies (6.12). Finally, (6.11) follows from (6.13) and (6.12).

Continuing with the proof of Theorem [1.5, it follows from (6.6), (6.12), and (6.8) that

$$
\int_{|y|<1}|y|\left(-\Delta^{2} u(y)\right) d y<\infty
$$

Since, by (6.14), (3.3), and (3.2),

$$
\begin{aligned}
\infty>\int_{|x|<1}|x|\left(-\Delta^{2} u(x)\right) d x & \geq \int_{S_{1}(0)}|x||x|^{\sigma-7} f\left(|x|^{-1} u(x)\right) d x \\
& \geq \frac{1}{M^{1-\sigma / 2}} \int_{S_{1}(0)}|x|^{-4}\left(M|x|^{-2}\right)^{1-\sigma / 2} f\left(M|x|^{-2}\right) d x \\
& \geq \frac{1}{M^{1-\sigma / 2}}\left(\min _{t \geq M} t^{1-\sigma / 2} f(t)\right) \int_{S_{1}(0)}|x|^{-4} d x,
\end{aligned}
$$

it follows from (1.15) that $\int_{S_{1}(0)}|x|^{-4} d x<\infty$. Hence

$$
\int_{|x|<1}|x|^{-3} u(x) d x \leq M \int_{S_{1}(0)}|x|^{-4} d x+\int_{B_{1}(0) \backslash S_{1}(0)}|x|^{-2} d x<\infty .
$$

Thus by Lemma 2.7 we have

$$
\int_{|y|<1}-\Delta^{2} u(y) d y<\infty
$$

Equation (6.15) also implies

$$
\int_{|x|<r} u(x) d x \leq r^{3} \int_{|x|<r}|x|^{-3} u(x) d x=o\left(r^{3}\right) \quad \text { as } \quad r \rightarrow 0^{+} .
$$


Let $F=\Delta^{2} u$ and

$$
\hat{N}(x)=\int_{B_{1}(0)} \Phi(x-y) F(y) d y \quad \text { for } \quad x \in \mathbb{R}^{3} .
$$

It follows from (1.12) and (6.16) that $\hat{N} \in C^{1}\left(\mathbb{R}^{3}\right)$. In particular

$$
U(x):=\hat{N}(x)-\hat{N}(0)-D \hat{N}(0) x=o(|x|) \quad \text { as } x \rightarrow 0 .
$$

Also for $\varphi \in C_{0}^{\infty}\left(B_{1}(0)\right)$ we have

$$
\begin{aligned}
\int_{B_{1}(0)} \hat{N} \Delta^{2} \varphi d x & =\int_{B_{1}(0)}\left(\int_{B_{1}(0)} \Phi(x-y) \Delta^{2} \varphi(x) d x\right) F(y) d y \\
& =\int_{B_{1}(0)} \varphi(y) F(y) d y .
\end{aligned}
$$

Thus $F=\Delta^{2} \hat{N}=\Delta^{2} U$ in $\mathcal{D}^{\prime}\left(B_{1}(0)\right)$.

Let $v=u-U$. By (6.17) and (6.18) we see that $v$ satisfies (2.7). Since $\Delta^{2} u=F$ in $\mathcal{D}^{\prime}\left(B_{1}(0) \backslash\right.$ $\{0\})$ we have $\Delta^{2} v=\Delta^{2} u-\Delta^{2} U=0$ in $\mathcal{D}^{\prime}\left(B_{1}(0) \backslash\{0\}\right)$. Thus Lemma 2.5 implies for some constant $b_{0}$ and some $C^{\infty}$ solution $H$ of $\Delta^{2} H=0$ in $B_{1}(0)$ we have

$$
\begin{aligned}
u & =U(x)+b_{0}|x|+H(x) \quad \text { in } \quad B_{1}(0) \backslash\{0\} \\
& =b_{0}|x|+H(x)+o(|x|) \quad \text { as } x \rightarrow 0
\end{aligned}
$$

by (6.18). Hence the positivity of $u$ implies $H(0) \geq 0$. If $H(0)>0$ then by (6.20) we have $u(x)>\varepsilon>0$ for $|x|$ small and positive which contradicts (6.17). Thus $H(0)=0$. Hence (6.20) implies

$$
u(x)=O(|x|) \quad \text { as } x \rightarrow 0
$$

and the biharmonicity of $H$ implies

$$
\bar{H}(r)=-b_{1} A r^{2} \quad \text { for some constant } b_{1}
$$

where $\bar{H}$ is the average of $H$ on the sphere $|x|=r$. By (6.21) and (3.3) we see for some positive constant $C$ that

$$
-\Delta^{2} u(x) \geq C|x|^{2 \sigma-12} u(x)^{5-\sigma} \quad \text { for } \quad 0<|x| \leq 1 .
$$

Averaging (6.20) we find by (6.22) that

$$
0<\bar{u}(r)=b_{0} r+o(r) \quad \text { as } r \rightarrow 0 .
$$

Hence $b_{0} \geq 0$. If $b_{0}>0$ then for some constant $C>0$ we have $\bar{u}(r) \geq C r$ for $0<r \leq 1$ and thus averaging (6.23) we get $-\overline{\Delta^{2} u}(r) \geq C r^{\sigma-7} \geq C r^{-5}$ for $0<r \leq 1$ which contradicts (6.16). Hence $b_{0}=0$ and so averaging (6.19) and using (6.22) we get

$$
\frac{\bar{u}(r)}{A}=\frac{\bar{U}(r)}{A}-b_{1} r^{2} \quad \text { for } 0<r \leq 1 .
$$

It follows from (6.18) and (6.1) that for $0<r<1$ we have

$$
\begin{aligned}
\frac{1}{A} \bar{U}(r) & =\frac{1}{A} \overline{(\hat{N}-\hat{N}(0))}(r) \\
& =\int_{|y|<1}\left(\frac{1}{\left|\partial B_{r}\right|} \int_{|x|=r}|x-y| d S_{x}-|y|\right)\left(-\Delta^{2} u(y)\right) d y \\
& =\int_{|y|<r}\left(r-|y|+\frac{1}{3} r^{-1}|y|^{2}\right)\left(-\Delta^{2} u(y)\right) d y+\int_{r<|y|<1} \frac{1}{3} r^{2}|y|^{-1}\left(-\Delta^{2} u(y)\right) d y .
\end{aligned}
$$


Hence by (6.24),

$$
\frac{\bar{u}(r)}{A}=\int_{|y|<r} r\left[1-\frac{|y|}{r}+\frac{1}{3}\left(\frac{|y|}{r}\right)^{2}\right]\left(-\Delta^{2} u(y)\right) d y+\left(J(r)-b_{1}\right) r^{2}
$$

where

$$
J(r):=\int_{r<|y|<1} \frac{1}{3}|y|^{-1}\left(-\Delta^{2} u(y)\right) d y \quad \text { for } \quad 0 \leq r<1
$$

$(J(0)$ may be $\infty$.)

Case I. Suppose $b_{1}<J(0)$. Then there exists $\varepsilon>0$ and $r_{0} \in(0,1)$ such that $b_{1} \leq(1-\varepsilon) J(r)$ for

$0<r \leq r_{0}$. Thus by (6.25), $\frac{\bar{u}(r)}{A} \geq \varepsilon r^{2} J(r)$ for $0<r<r_{0}$, which together with (6.23) and Lemma 2.6 gives a contradiction and thereby proves Theorem 1.5] when $n=3$ in Case I.

Case II. Suppose $b_{1} \geq J(0)$. Then for $0<r<1$ we have

$$
\left(J(r)-b_{1}\right) r^{2} \leq(J(r)-J(0)) r^{2}=-\int_{|y|<r} \frac{1}{3} r^{2}|y|^{-1}\left(-\Delta^{2} u(y)\right) d y
$$

and hence by (6.25) we have

$$
\begin{aligned}
0<\frac{\bar{u}(r)}{A} & \leq \int_{|y|<r} r\left[1-\frac{|y|}{r}+\frac{1}{3}\left(\left(\frac{|y|}{r}\right)^{2}-\frac{r}{|y|}\right)\right]\left(-\Delta^{2} u(y)\right) d y \\
& =-\frac{1}{3} \int_{|y|<r}|y|^{2} r^{-1}\left(\frac{r}{|y|}-1\right)^{3}\left(-\Delta^{2} u(y)\right) d y .
\end{aligned}
$$

This contradiction completes the proof of Theorem 1.5] in all cases.

\section{Proof of Theorem 1.6}

In this section we prove Theorem 1.6.

Proof of Theorem 1.6. Suppose for contradiction that $v(y)$ is a $C^{4}$ positive solution of (1.14) in $\mathbb{R}^{2} \backslash \Omega$. By scaling $v$, we can assume $\Omega=\overline{B_{1 / 2}(0)}$. Let $u(x)=|y|^{-2} v(y), y=\frac{x}{|x|^{2}}$ be the 2-Kelvin transform of $v(y)$. Then

$$
v(y)=|x|^{-2} u(x) \quad \text { and } \quad \Delta^{2} v(y)=|x|^{6} \Delta^{2} u(x) .
$$

It follows therefore from (1.14) that $u(x)$ is a $C^{4}$ positive solution of

$$
-\Delta^{2} u(x) \geq|x|^{\sigma-6} f\left(|x|^{-2} u(x)\right) \quad \text { in } B_{2}(0) \backslash\{0\} .
$$

Choose $s_{0}>1$ and so large that the function

$$
g(s):= \begin{cases}\frac{(\log s)^{1-\sigma / 2}}{s^{1-\sigma / 2} \prod_{i=2}^{k} \log ^{i} s} & \text { if } s \geq s_{0} \\ g\left(s_{0}\right) & \text { if } 0<s<s_{0}\end{cases}
$$

is well defined, continuous, positive, and nonincreasing for $s>0$. By (1.16) we have for some positive constant $C$ that

$$
f(s) \geq C g(s) \text { for } \quad s \geq 1 .
$$


Since $u$ is a $C^{4}$ positive solution of (A.2) it follows from Theorem A.1 that $u$ satisfies (A.4) and, for some constant $M>e$,

$$
u(x) \leq M \log \frac{e}{|x|} \quad \text { for } \quad 0<|x| \leq 1 .
$$

Since

$$
\frac{g\left(M|x|^{-2} \log \frac{e}{|x|}\right)}{|x|^{2-\sigma} / \prod_{i=2}^{k} \log ^{i} \frac{1}{|x|}} \rightarrow\left(\frac{2}{M}\right)^{1-\sigma / 2} \quad \text { as } \quad x \rightarrow 0
$$

there exists $r_{0} \in\left(0,1 / s_{0}\right)$ such that

$$
g\left(M|x|^{-2} \log \frac{e}{|x|}\right) \geq \frac{|x|^{2-\sigma}}{M^{1-\sigma / 2} \prod_{i=2}^{k} \log ^{i} \frac{1}{|x|}} \quad \text { for } 0<|x|<r_{0} .
$$

Let $D=\left\{x \in B_{r_{0}}(0) \backslash\{0\}:|x|^{2} \leq u(x) \leq M \log \frac{e}{|x|}\right\}$. Since $1 \leq|x|^{-2} u(x) \leq M|x|^{-2} \log \frac{e}{|x|}$ for $x \in D$, it follows from (7.2), (7.3), and (7.5) that for $\alpha>0$ we have

$$
\begin{aligned}
I(\alpha) & :=\int_{D}|x|^{\alpha}\left(-\Delta^{2} u(x)\right) d x \\
& \geq \int_{D}|x|^{\alpha}|x|^{\sigma-6} f\left(|x|^{-2} u(x)\right) d x \\
& \geq C \int_{D}|x|^{\alpha+\sigma-6} g\left(M|x|^{-2} \log \frac{e}{|x|}\right) d x \\
& \geq C \int_{D} \frac{|x|^{\alpha-4}}{\prod_{i=2}^{k} \log ^{i} \frac{1}{|x|}} d x .
\end{aligned}
$$

Hence by (7.4)

$$
\begin{aligned}
\int_{|x|<r_{0}} \frac{|x|^{\alpha-4}}{\log \frac{e}{|x|} \prod_{i=2}^{k} \log ^{i} \frac{1}{|x|}} u(x) d x & \leq M \int_{D} \frac{|x|^{\alpha-4}}{\prod_{i=2}^{k} \log ^{i} \frac{1}{|x|}} d x+\int_{B_{r_{0}}(0) \backslash D} \frac{|x|^{\alpha-2}}{\log \frac{e}{|x|} \prod_{i=2}^{k} \log ^{i} \frac{1}{|x|}} d x \\
& \leq C I(\alpha)+\int_{B_{r_{0}}(0)} \frac{|x|^{\alpha-2}}{\log \frac{e}{|x|} \prod_{i=2}^{k} \log ^{i} \frac{1}{|x|}} d x .
\end{aligned}
$$

By (A.4), $I(2)<\infty$. Thus (7.6) with $\alpha=2$ and Lemma 2.7 imply

$$
\int_{|x|<1}-\Delta^{2} u(x) d x<\infty .
$$

Hence $I(1 / 2)<\infty$ and thus by (7.6) with $\alpha=1 / 2$ we have

$$
\frac{1}{r^{7 / 2} \log \frac{e}{r} \prod_{i=2}^{k} \log ^{i} \frac{1}{r}} \int_{|x|<r} u(x) d x=o(1) \quad \text { as } \quad r \rightarrow 0^{+} .
$$

Therefore

$$
\int_{|x|<r} u(x) d x=o\left(r^{3}\right) \quad \text { as } \quad r \rightarrow 0^{+}
$$

Let $F=\Delta^{2} u$ and

$$
N(x)=\int_{|y|<1} \Phi(x-y) F(y) d y \quad \text { for } \quad x \in \mathbb{R}^{2}
$$


where $\Phi$ is the fundamental solution of $\Delta^{2}$ in $\mathbb{R}^{2}$ given by (1.13). It follows from (7.7) and (1.13) that $N \in C^{1}\left(\mathbb{R}^{2}\right)$. In particular

$$
U(x):=N(x)-N(0)-(D N)(0) x=o(|x|) \quad \text { as } \quad x \rightarrow 0 .
$$

Also for $\varphi \in C_{0}^{\infty}\left(B_{1}(0)\right)$ we have

$$
\begin{aligned}
\int_{B_{1}(0)} N \Delta^{2} \varphi d x & =\int_{B_{1}(0)}\left(\int_{B_{1}(0)} \Phi(x-y) \Delta^{2} \varphi(x) d x\right) F(y) d y \\
& =\int_{B_{1}(0)} \varphi(y) F(y) d y .
\end{aligned}
$$

Thus $F=\Delta^{2} N=\Delta^{2} U$ in $\mathcal{D}^{\prime}\left(B_{1}(0)\right)$.

Let $v=u-U$. By (7.8) and (7.9) we see that $v$ satifies (2.7). Since $\Delta^{2} u=F$ in $\mathcal{D}^{\prime}\left(B_{1}(0) \backslash\{0\}\right)$ we have $\Delta^{2} v=\Delta^{2} u-\Delta^{2} U=0$ in $\mathcal{D}^{\prime}\left(B_{1}(0) \backslash\{0\}\right)$. Thus Lemma 2.5 implies for some constant $b$ that

$$
u(x)=U(x)+b|x|^{2} \log \frac{e}{|x|}+H(x) \quad \text { in } \quad B_{1}(0) \backslash\{0\}
$$

where $H$ is a $C^{\infty}$ biharmonic function in $B_{1}(0)$.

It follows from (7.10), (7.9), and the positivity of $u$ that $H(0) \geq 0$. If $H(0)>0$ then, by (7.10) and (7.9),$u(x)>\varepsilon>0$ for $|x|$ small and positive, which contradicts (7.8). Thus $H(0)=0$. Hence by (7.10), (7.9), and the positivity of $u$ we have $D H(0)=0$ and thus

$$
H(x)=O\left(|x|^{2}\right) \quad \text { as } \quad x \rightarrow 0 .
$$

For $x \in \mathbb{R}^{2}$ we see by Lemma 2.1 that

$$
\frac{1}{\left|\partial B_{r}\right|} \int_{|y|=r}|x-y|^{2} \log \frac{e}{|x-y|} d S_{y}= \begin{cases}r^{2} \log \frac{e}{r}+|x|^{2} \log \frac{1}{r}, & \text { if }|x|<r \\ |x|^{2} \log \frac{e}{|x|}+r^{2} \log \frac{1}{|x|}, & \text { if }|x|>r .\end{cases}
$$

It therefore follows from (7.9) and (7.7) that for $0<r<e^{-1}$ we have

$$
\begin{aligned}
\left|\frac{1}{A} \bar{U}(r)\right|= & \left|\frac{1}{A} \overline{(N-N(0))}(r)\right| \\
= & \left|\int_{|y|<1} \frac{1}{\left|\partial B_{r}\right|} \int_{|x|=r}\left(\frac{-\Phi(x-y)}{A}+\frac{\Phi(y)}{A}\right) d S_{x}\left(-\Delta^{2} u(y)\right) d y\right| \\
= & \left|\int_{|y|<1}\left(\frac{1}{\left|\partial B_{r}\right|} \int_{|x|=r}|x-y|^{2} \log \frac{e}{|x-y|} d S_{x}-|y|^{2} \log \frac{e}{|y|}\right)\left(-\Delta^{2} u(y)\right) d y\right| \\
= & \mid \int_{|y|<r}\left(r^{2} \log \frac{e}{r}+|y|^{2} \log \frac{1}{r}-|y|^{2} \log \frac{e}{|y|}\right)\left(-\Delta^{2} u(y)\right) d y \\
& +\int_{r<|y|<1}\left(r^{2} \log \frac{1}{|y|}\right)\left(-\Delta^{2} u(y)\right) d y \mid \\
\leq & r^{2} \log \frac{1}{r} \int_{r<|y|<\left(\log \frac{1}{r}\right)^{-1}}-\Delta^{2} u(y) d y+r^{2} \log \log \frac{1}{r} \int_{|y|<1}-\Delta^{2} u(y) d y \\
& +r^{2} \log \frac{e}{r} \int_{|y|<r}\left|1+\frac{e^{2}\left(\frac{|y|}{e r}\right)^{2} \log \frac{|y|}{e r}}{\log \frac{e}{r}}\right|\left(-\Delta^{2} u(y)\right) d y \\
= & o\left(r^{2} \log \frac{e}{r}\right) \quad \text { as } \quad r \rightarrow 0^{+} .
\end{aligned}
$$


Thus averaging (17.10) and noting (7.11) we get

$$
\bar{u}(r)=b r^{2} \log \frac{e}{r}+o\left(r^{2} \log \frac{e}{r}\right) \quad \text { as } \quad r \rightarrow 0^{+}
$$

which together with the positivity of $u$ implies

$$
b \geq 0 .
$$

It follows from the integral form of the remainder in Taylor's theorem that if $x, y \in B_{1}(0) \backslash\{0\}$ are such that $t x-y \neq 0$ for all $t \in[0,1]$ then

$$
\Phi(x-y)-\Phi(-y)-D \Phi(-y) x=-2 A|x|^{2} \int_{0}^{1}(1-t) \log \frac{e}{|t x-y|} d t+\hat{\Phi}(x, y)
$$

where

$$
|\hat{\Phi}(x, y)|=A\left|\frac{|x|^{2}}{2}+2 \int_{0}^{1}(1-t) \frac{[(t x-y) \cdot x]^{2}}{|t x-y|^{2}} d t\right| \leq \frac{3}{2} A|x|^{2} .
$$

Thus for $x \in B_{1}(0) \backslash\{0\}$ we have

$$
U(x)=2 A|x|^{2} \int_{|y|<1} \int_{0}^{1}(1-t) \log \frac{e}{|t x-y|} d t\left(-\Delta^{2} u(y)\right) d y+O\left(|x|^{2}\right)
$$

by (7.7).

By Fatou's lemma,

$$
\liminf _{x \rightarrow 0} \int_{|y|<1} \int_{0}^{1}(1-t) \log \frac{e}{|t x-y|} d t\left(-\Delta^{2} u(y)\right) d y \geq \frac{1}{2} \int_{|y|<1} \log \frac{e}{|y|}\left(-\Delta^{2} u(y)\right) d y .
$$

Case I. Suppose $\int_{|y|<1}\left(\log \frac{e}{|y|}\right)\left(-\Delta^{2} u(y)\right) d y=\infty$. Then it follows from (7.13), (7.14), (7.12), (7.11), and (7.10) that $u(x)>>|x|^{2}$ as $x \rightarrow 0$. Thus, reversing the original change of variables (7.1), we have $v(y)>1$ for $|y| \geq r_{0} / 2$ for some $r_{0}>2$ and $v(y)$ is a solution of

$$
-\Delta^{2} v \geq|y|^{-\sigma} f(v) \geq C|y|^{-\sigma} g(v) \geq C|y|^{-\sigma} v^{-1+\sigma / 2} \quad \text { in } \mathbb{R}^{2} \backslash B_{r_{0} / 2}(0)
$$

where $C$ is a positive constant and $g$ is the function in (7.3). Averaging (7.15) we see that $\bar{v}(r)$ is a positive radial solution of $-\Delta^{2} \bar{v} \geq C|y|^{-\sigma} \bar{v}^{-1+\sigma / 2}$ in $\mathbb{R}^{2} \backslash B_{r_{0} / 2}(0)$ which contradicts Lemma 2.8 and completes the proof of Theorem 1.6 in Case I.

Case II. Suppose $\int_{|y|<1}\left(\log \frac{e}{|y|}\right)\left(-\Delta^{2} u(y)\right) d y<\infty$. Then since

$$
\log \frac{e}{|t x-y|}=\log \frac{e}{|y|}+\log \frac{|y|}{|y-t x|}
$$

we see that (17.13) and Lemma 2.9 imply $U(x)=O\left(|x|^{2}\right)$ as $x \rightarrow 0$. Hence if $b>0$ (resp. $\left.b=0\right)$ then it follows from (7.10) and (7.11) that

$$
u(x)>>|x|^{2} \quad\left(\text { resp. } u(x)=O\left(|x|^{2}\right)\right) \quad \text { as } \quad x \rightarrow 0 .
$$

If $u(x)>>|x|^{2}$ as $x \rightarrow 0$ then we obtain a contradiction as in Case I. Thus we can assume for some $s_{0}>0$ that $|x|^{-2} u(x)<s_{0}$ for $0<|x| \leq 1$. Hence reversing the original change of variables (7.1) we get

$$
0<v(y)<s_{0} \quad \text { for } \quad|y| \geq 1
$$


and $v(y)$ is a solution of

$$
-\Delta^{2} v \geq|y|^{-\sigma} f(v) \text { in } \quad \mathbb{R}^{2} \backslash B_{1}(0) .
$$

We can assume $\left.f\right|_{\left(0, s_{0}\right]}$ is $C^{2}$ and $\left(\left.f\right|_{\left(0, s_{0}\right]}\right)^{\prime \prime}>0$ because, as one easily verifies, the function $\hat{f}$ : $\left(0, s_{0}\right] \rightarrow(0, \infty)$ defined by

$$
\hat{f}(s)=\frac{1}{s_{0}^{2}} \int_{0}^{s}(s-\zeta)\left(\min _{\zeta \leq \tau \leq s_{0}} f(\tau)\right) d \zeta
$$

is $C^{2}$ and satisfies

$$
0<\hat{f}(s) \leq f(s) \text { and } \quad \hat{f}^{\prime \prime}(s)>0 \text { for } 0<s \leq s_{0} .
$$

Thus averaging (17.17) and noting (17.16) we find that $\bar{v}(r)$ is a positive solution of $-\Delta^{2} \bar{v} \geq|y|^{-\sigma} f(\bar{v})$ in $\mathbb{R}^{2} \backslash B_{1}(0)$ which contradicts Lemma 2.8 and completes the proof of Theorem 1.6 in all cases.

\section{A Represention formula and pointwise bound}

Let $\Phi$ be the fundamental solution of $\Delta^{2}$ in $\mathbb{R}^{n}$ given by (1.10) $-(1.13)$ and for $x \neq 0$ and $y \neq x$, let

$$
\Psi(x, y)=\Phi(x-y)-\sum_{|\beta| \leq 1} \frac{(-y)^{\beta}}{\beta !} D^{\beta} \Phi(x)
$$

be the error in approximating $\Phi(x-y)$ with the partial sum of degree one of the Taylor series of $\Phi$ at $x$.

The following theorem, which we proved in [9], gives representation formula (A.5) and pointwise bound (A.3) for nonnegative solutions of

$$
-\Delta^{2} u \geq 0 \quad \text { in } \quad B_{2}(0) \backslash\{0\} \subset \mathbb{R}^{n} .
$$

See [6] and [7] for some similar results.

Theorem A.1. Let $u(x)$ be a $C^{4}$ nonnegative solution of (A.2) where $n \geq 2$. Then

$$
\begin{aligned}
u(x)= & \left\{\begin{array}{ll}
O\left(|x|^{2-n}\right) & \text { if } n \geq 3 \\
O\left(\log \frac{e}{|x|}\right) & \text { if } n=2
\end{array} \text { as } \quad x \rightarrow 0,\right.
\end{aligned}
$$

and

$$
u=N+h+\sum_{|\beta| \leq 2} a_{\beta} D^{\beta} \Phi \quad \text { in } \quad B_{1}(0) \backslash\{0\},
$$

where $a_{\beta},|\beta| \leq 2$, are constants, $h \in C^{\infty}\left(\overline{B_{1}(0)}\right)$ is a solution of $\Delta^{2} h=0$ in $\overline{B_{1}(0)}$, and

$$
N(x)=\int_{|y|<1} \Psi(x, y) \Delta^{2} u(y) d y \quad \text { for } \quad x \neq 0 .
$$

Acknowledgement. We would like to thank Guido Sweers for helpful comments. 


\section{References}

[1] Armstrong, S., Sirakov, B. (2011). Nonexistence of positive supersolutions of elliptic equations via the maximum principle. Comm. Partial Differential Equations 36:2011-2047.

[2] Brezis, H., Lions, P.L. (1981). A note on isolated singularities for linear elliptic equations. Mathematical analysis and applications, Part A, pp. 263-266, Adv. in Math. Suppl. Stud., 7a, Academic Press, New York-London.

[3] Caristi, G., D'Ambrosio, L., Mitidieri, E. (2008). Representation formulae for solutions to some classes of higher order systems and related Liouville theorems. Milan J. Math. 76:27-67.

[4] Caristi, G., Mitidieri, E., Soranzo, R. (1998). Isolated singularities of polyharmonic equations. Atti Sem. Mat. Fis. Univ. Modena 46:257-294.

[5] Choi, Y.S., Xu, X. (2009). Nonlinear biharmonic equations with negative exponents. J. Differential Equations 246:216-234.

[6] Futamura, T., Kishi, K., Mizuta, Y. (2003). Removability of sets for sub-polyharmonic functions. Hiroshima Math. J. 33:31-42.

[7] Futamura, T., Mizuta, Y. (2004). Isolated singularities of super-polyharmonic functions. Hokkaido Math. J. 33:675-695.

[8] Gazzola, F., Grunau, H.-C., Sweers, G. (2010). Polyharmonic Boundary Value Problems, Springer.

[9] Ghergu, M., Moradifam, A., Taliaferro, S. (2011). Isolated singularities of polyharmonic inequalities. J. Funct. Anal. 261:660-680.

[10] Guerra, I. (2012). A note on the nonlinear biharmonic equations with negative exponents, $J$. Differential Equations, 253:3147-3157.

[11] Guo, Y., Liu, J. (2008). Liouville-type theorems for polyharmonic equations in $\mathbb{R}^{N}$ and in $\mathbb{R}_{+}^{N}$. Proc. Roy. Soc. Edinburgh Sect. A 138:339-359.

[12] Hsu, S.-Y. (2010). Removable singularity of the polyharmonic equation. Nonlinear Anal. $72: 624-627$.

[13] McKenna, P.J., Reichel, W. (2003). Radial solutions of singular nonlinear biharmonic equations and applications to conformal geometry. Electron. J. Differential Equations. 37:13 pp.

[14] Mitidieri, E., Pohozaev, S.I. (2001). Apriori Estimates and Blow-up of Solutions to Nonlinear Partial Differential Equations and Inequalities.

[15] Taliaferro, S. (in press). Pointwise Bounds and Blow-up for Nonlinear Polyharmonic Inequalities. Ann. Inst. H. Poincare Anal. Non lineaire.

[16] Wei, J., Xu, X. (1999). Classification of solutions of higher order conformally invariant equations. Math. Ann. 313:207-228.

[17] Xu, X. (2000). Uniqueness theorem for the entire positive solutions of biharmonic equations in $R^{n}$. Proc. Roy. Soc. Edinburgh Sect. A 130:651-670. 